\title{
Optimization of quasi-axisymmetric stellarators with varied elongation
}

\author{
Zhichen Feng ${ }^{1}$, David A. Gates ${ }^{2}$, Samuel A. Lazerson ${ }^{2,3}$, \\ Matt Landreman ${ }^{4}$, Neil Pomphrey ${ }^{2}$, and GuoYong $\mathrm{Fu}^{1 *}$ \\ ${ }^{1}$ Institute for Fusion Theory and Simulation and Department of physics, \\ Zhejiang University, Hangzhou 310027, China \\ ${ }^{2}$ Princeton Plasma Physics Laboratory, Princeton, New Jersey 08543, USA \\ ${ }^{3}$ Max-Planck-Institut für Plasmaphysik, Greifswald, 17491, Germany and \\ ${ }^{4}$ Institute for Research in Electronics and Applied Physics, \\ University of Maryland, College Park, Maryland, 20742, USA
}

\begin{abstract}
An optimization study of Quasi-Axisymmetric (QA) stellarators with varied elongation has been carried out using the optimization code STELLOPT. The starting point of our optimization is a previously obtained QA stellarator with 3 field periods and aspect ratio of 6 . A series of QA stellarators are obtained at zero plasma beta with varied elongation value ranging from 2.5 to 3.7 . Good quasi-symmetry is kept when the elongation value is reduced from the original value of 3.7. The rotational transform profile and aspect ratio are kept fixed. The plasma volume is ether kept fixed or varied linearly with elongation. Furthermore, finite beta QA stellarators are considered. The corresponding bootstrap currents are calculated using the kinetic code SFINCS. A series of kink-stable QA stellarators are obtained via optimization with varied plasma beta up to $5 \%$ and self-consistent bootstrap current. This work demonstrates that good QA stellarators with finite beta and varied elongation exist that are stable to external kink modes.
\end{abstract}

*corresponding author’s Email: gyfu@zju.edu.cn 


\section{INTRODUCTION}

Quasi symmetry has been proven to be a powerful concept for improving neoclassical transport in stellarators. The idea came from the original work of Boozer[1] who showed that the particle orbits in 3D stellarator geometry only depend on the magnitude of magnetic field in Boozer coordinates. Thus the particle orbits in stellarators are equivalent to those of tokamaks if the magnetic field strength is symmetric in Boozer coordinates even though the vector magnetic field and geometry of magnetic surfaces are 3D. Using this idea Nührenberg and Zille[2] demonstrated numerically the existence of quasi-helically symmetric $(\mathrm{QH})$ stellarators. $\mathrm{QH}$ means that in the Fourier expansion of the magnitude of magnetic field on a magnetic flux, $B=\sum_{m, n} B_{m, n} \cos (m \theta-n \phi)$, the dominant Fourier components $B_{m, n}$ are of only one $m / n$ ratio, where $\theta$ and $\phi$ are poloidal and toroidal Boozer coordinates respectively. This led to the Helical Symmetric eXperiment (HSX) which validated the theory of QH experimentally[3]. Furthermore the existence of Quasi AxiSymmetric (QA) stellarators was also shown[4,5], where QA means that the dominant Fourier components $B_{m, n}$ are only of $n=0$. Since then a number of QA stellarators have been designed including NCSX[6], CHS-qa[7], ESTELL[8], and more recently, CFQS[9] and the new QA stellarator by Henneberg et al.[10]. In particular, CFQS is currently being built in China. A reactor concept based on an optimized QA configuration has also been designed[11]. Compared to tokamak reactors, QA stellarators have advantages of steady state operation without external current drive and potentially disruption free operation. The freedom of 3D geometry also allows more external control over important properties such as MHD stability and plasma transport while maintaining quasisymmetry[12].

In this work we have carried out an optimization study of QA stellarators with varied elongation. It is known that, for tokamaks, the elongation has strong effects on MHD stability[13] and plasma transport[14]. Thus it is important to investigate the dependence of QA stellarators on elongation. The present work starts from a 3 field period aspect ratio $=6$ QA configuration obtained by $\mathrm{Ku}[15]$ who made a systematic study of the dependence of modular coil geometry on aspect ratio, number of field periods, external rotational transform and plasma-coil spacing. Our work here, on the other hand, is focused on the effects of elongation. This work extends the previous work to a range of elongation at both zero beta and finite beta with self-consistent bootstrap current. The elongation scan is carried out both at a fixed plasma volume and with volume proportional to elongation. The latter scan is motivated by the fact that for axisymmetric tokamaks the plasma volume is proportional to elongation at fixed major radius and minor radius. Our results demonstrate that good QA stellarators exist with a range of elongation that are stable to external kink modes. 
The paper is organized as follows. Sec II and III present results of an elongation scan at fixed volume and at volume proportional to elongation. Sec IV describes the calculation of self-consistent bootstrap current. Sec V presents the results of MHD stability optimization. Finally conclusions are given in Sec. VI.

\section{ELONGATION SCAN TARGETING CONSTANT VOLUME}

Here, we carry out an elongation scan starting from the 3 field period aspect ratio = 6 QA configuration obtained in the work by $\mathrm{Ku}$ and Boozer[15]. Elongation was not a target function in the original work, but rather an output quantity with plasma volume a constraint. We use the STELLOPT code[16] to do the optimization using the genetic algorithm with differential evolution (GADE) $[17,18]$ at each value of elongation in the zero beta case. In the optimization the sum of $\chi_{i}^{2}=\left(f_{i}^{\text {target }}-f_{i}\right)^{2} / \sigma_{i}^{2}$ is minimized, where $f_{i}$ is the ith variable chosen to be targeted, $f_{i}^{\text {target }}$ is its targeted value, and $\sigma_{i}$ is a weight parameter. We consider several critical physical properties listed in the following table.

TABLE I: Optimization targets when varying elongation at zero beta

\begin{tabular}{|c|c|c|c|}
\hline Target & Position & Targeted value & inverse weight $\sigma$ \\
\hline Elongation $\kappa$ & $\phi=0$ plane & $3.84-2.50$ & 0.01 \\
\hline$\epsilon_{\text {eff }}^{3 / 2}$ & $\mathrm{~s}=(2,16,32,64,127) / 128$ & 0 & 0.001 \\
\hline Helicity & $\mathrm{s}=(2,16,32,64,127) / 128$ & 0 & 0.05 \\
\hline Rotational transform $\iota$ & $\mathrm{s}=(2,64,127) / 128$ & $(0.257,0.277,0.313)$ & 0.001 \\
\hline Volume & entire volume & $444 \mathrm{~m}^{3}$ & 4 \\
\hline Aspect ratio & toroidally averaged & 6 & 0.1 \\
\hline
\end{tabular}

Here, the elongation $\kappa$ is defined by the shape of plasma boundary at the $\phi=0$ plane. Specifically $\kappa$ is the total height divided by the width at the mid-plane of the last closed flux surface; $\epsilon_{\text {eff }}^{3 / 2}$ is the effective helical ripple calculated by the NEO code[19]; $\mathrm{s}$ is the normalized toroidal flux; QA is enforced (helicity constraint) by assigning a target value of zero to each calculated non-axisymmetric component of field strength in Boozer Coordinates, where $B_{m, n}$ harmonics with $n=0-16$ and $|m|=0-32$ are included, and identical weights are assumed for each targeted harmonic. The weights are chosen by trial and error to give desirable results.

The following figures show the evolution of one optimization calculation targeting elongation to be $\kappa=3.64$ ( $\kappa=3.84$ is the initial configuration, from [15]). 


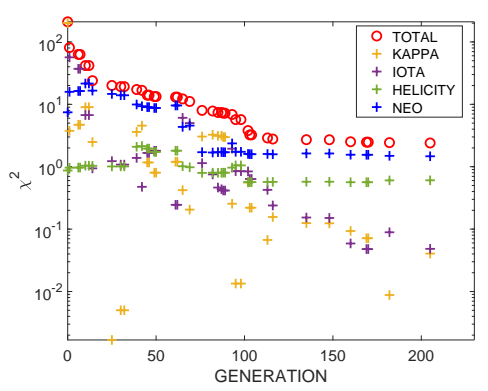

(a) $\chi^{2}$ evolution

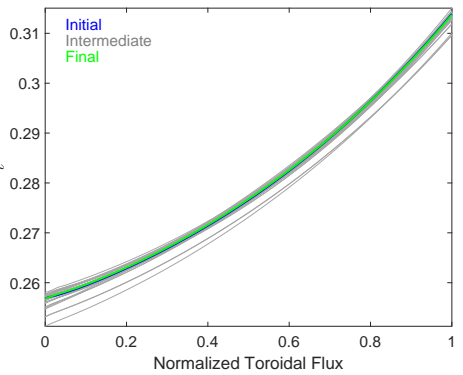

(c)Rotational transform evolution

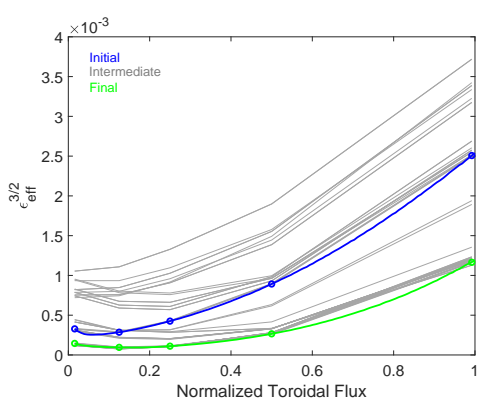

(b)Effective ripple evolution

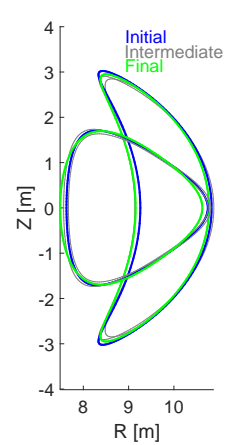

(d)Boundary flux surface evolution at $\phi=0$ and

$$
\phi=\pi / \mathrm{nfp}
$$

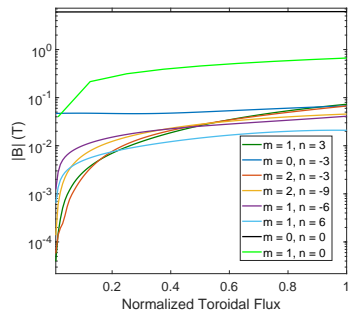

(e)Dominant $\left|B_{n m}\right|$ for the final case

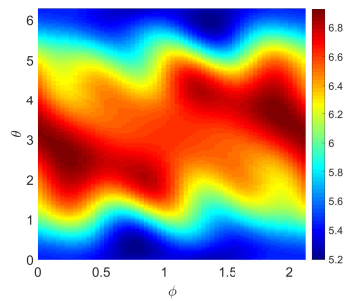

(f) $|B|$ on the boundary for the initial case

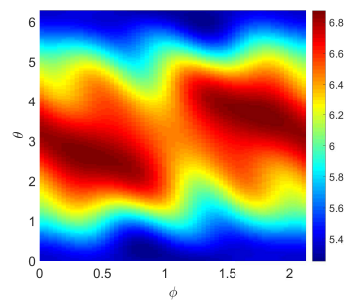

(g) $|B|$ on the boundary for the final case

FIG. 1: Progress of STELLOPT optimization using genetic algorithm with differential evolution targeting $\kappa=3.64$ 
Fig.1(a) shows the evolution of the total $\chi^{2}$ and its components. We observe that the total $\chi^{2}$ decreases to a constant value after approximately 100 iterations with $\chi_{i}^{2}$ of the neoclassical transport being the dominant component. Fig.1 (b), (c) and (d) show the evolution of effective ripple $\left(\epsilon_{e f f}^{3 / 2}\right)$ profile, the iota profile and the flux surface shape. We observe that the effective ripple decreases by a factor of two after optimization. The $\iota$ profile is almost identical to the initial one. The plasma boundary shape at $\phi=0$ shows a reduction of elongation to its target value of 3.64. Fig.1(e) plots the dominant Boozer $\left|B_{n m}\right|$ components as a function of normalized toroidal flux. It indicates that the optimized configuration is indeed a QA stellarator since the axi-symmetric components are much larger than the non-symmetric components. Fig.1(f) and $1(\mathrm{~g})$ show the $|B|$ patterns on the last closed flux surface for the initial and final cases. The results indicate that the magnetic field strength of the final case is nearly axi-symmetric and its symmetry is a little improved over that of the initial case.

The above optimization process is repeated for each target elongation value. This is done by decreasing the target value of elongation in each run by small increments. The results of this elongation scan are shown in Fig.2 which plots the flux surfaces for several representative values of elongation. Fig.3 shows the corresponding effective ripple $\left(\epsilon_{\text {eff }}^{3 / 2}\right)$ profile for the different elongation cases. The results suggest that the neoclassical transport can be kept at low levels when the elongation is reduced to lower values. It should be noted that the effective ripple level of the original configuration is significantly higher because it was optimized at finite plasma beta whereas here the zero beta case is considered. Table II shows the data of major physical quantities for different elongation cases after optimization, where $R_{\text {major }}$ is the averaged major radius calculated from volume and cross section, $a_{\text {minor }}$ is the averaged minor radius calculated from cross section area, $\langle B\rangle$ is the volume averaged magnetic field strength, and $B_{0}$ is the averaged magnetic field strength on axis. These quantities are kept almost the same for different elongation cases. This is achieved by targeting the aspect ratio, volume and the rotational transform to be the same as in the original case. The edge toroidal flux is held fixed during the optimization, leading to a slight variation in the magnetic field strength.

\section{ELONGATION SCAN TARGETING VOLUME PROPORTIONAL TO $\kappa$}

Here we carry out a different elongation scan at zero beta by targeting plasma volume proportional to $\kappa$ while other targets are kept the same as before. This is motivated by the fact that for axisymmetric tokamak geometry the volume is proportional to $\kappa$.

Fig. 4 and Fig.5 show the optimized outer boundary flux surfaces and the corresponding 


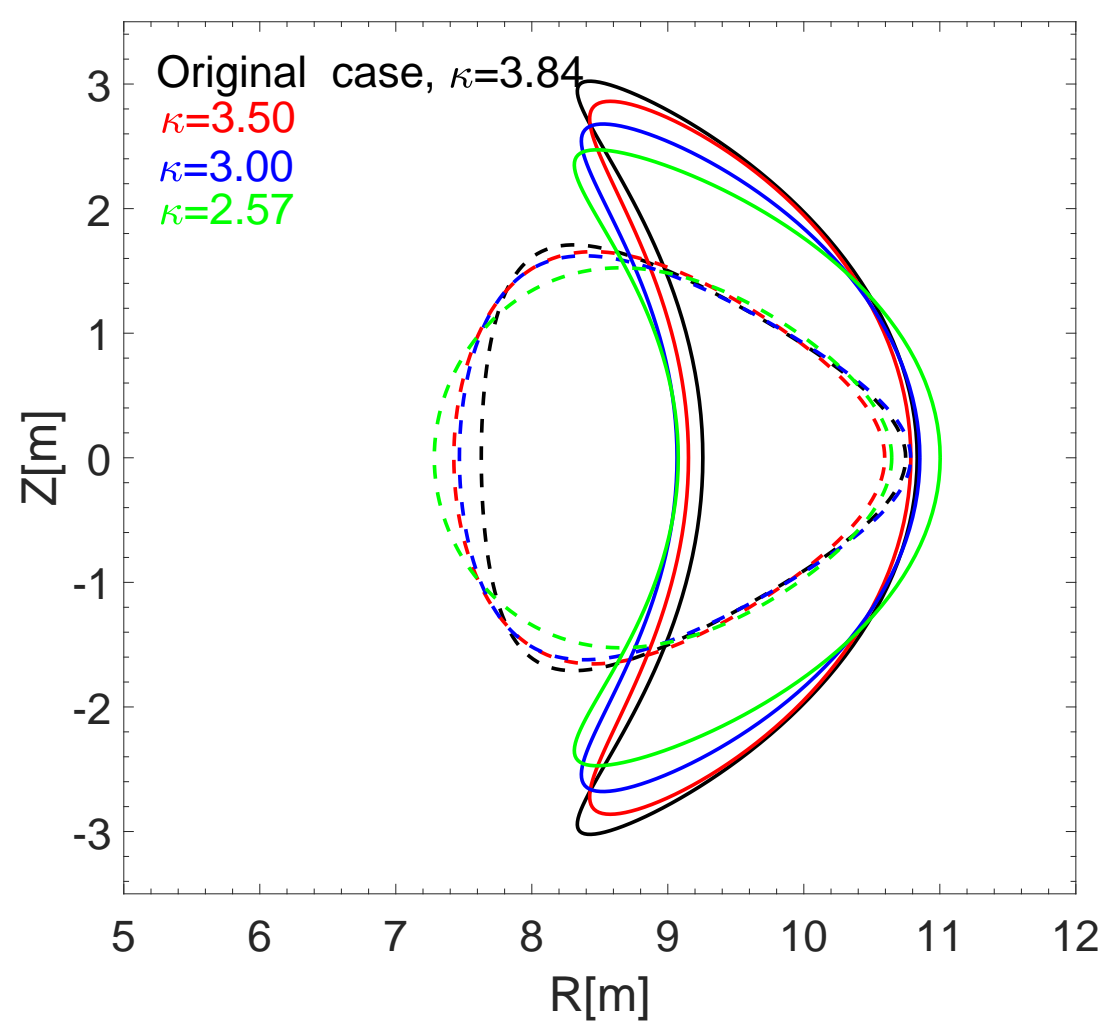

FIG. 2: Final boundary flux surface shapes as elongation is decreased

effective ripple $\left(\epsilon_{e f f}^{3 / 2}\right)$ profiles for several values of elongation. We see that the neoclassical transport levels are still kept at low levels.

There is a question on how to define $\kappa$ values for 3D stellarators. In this work we use $\kappa$ at $\phi=0$ plane as a measure of elongation. On the other hand, we can also use the averaged elongation, i.e., the elongation of the corresponding tokamak geometry defined by the $\mathrm{n}=0$ components of the 3D stellarator geometry. Fig. 6 plots the volume versus $R_{0} a_{0}^{2}\langle\kappa\rangle$ for stellarator and its corresponding tokamak. 


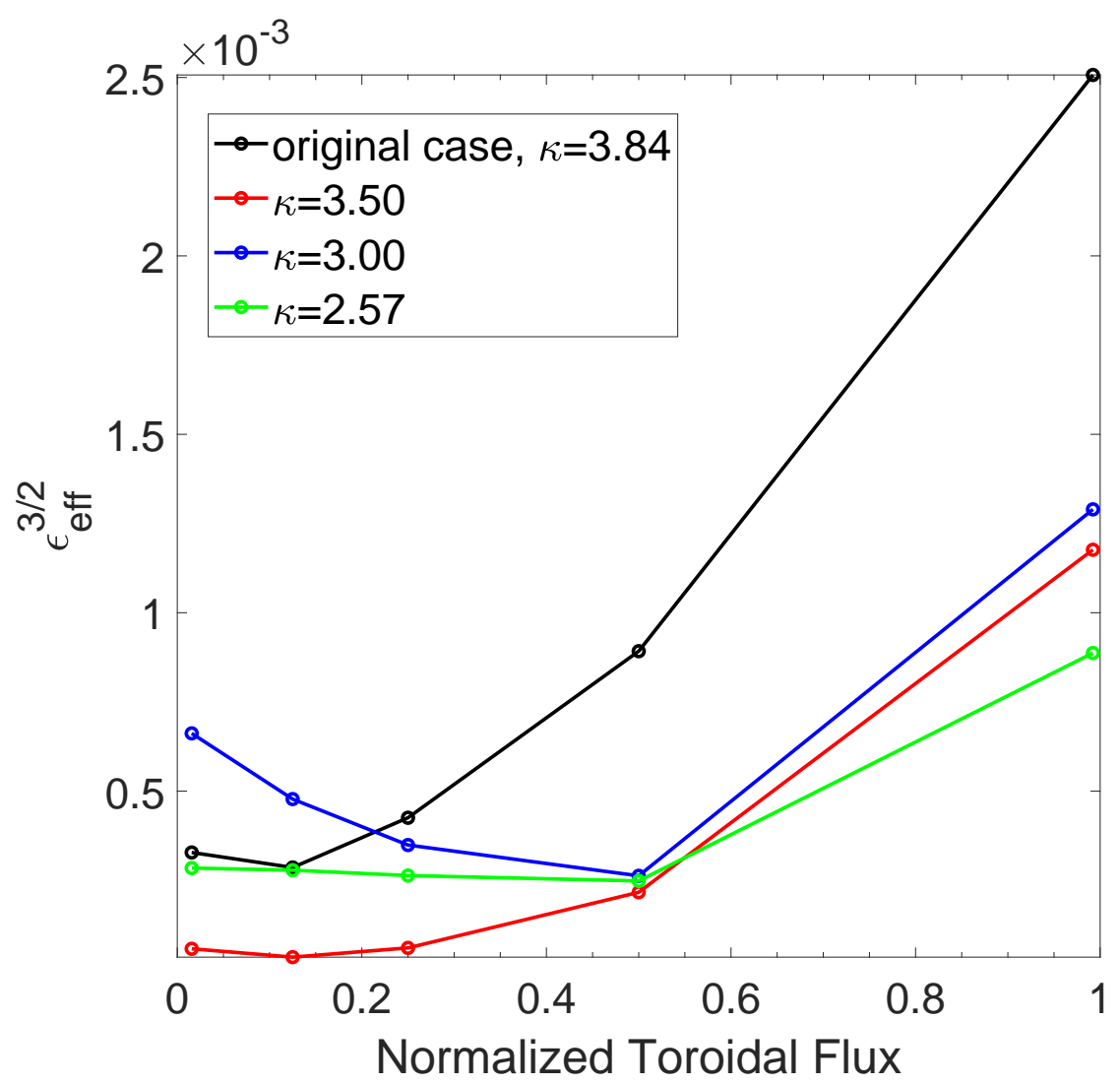

FIG. 3: Effective ripple variation when decreasing elongation

$R_{0}, a_{0},\langle\kappa\rangle$ is major radius, minor radius and elongation of the corresponding tokamak respectively. We observe that the volume of the corresponding tokamak is proportional to the product of $R_{0} a_{0}^{2}\langle\kappa\rangle$. The volume of stellarator is a little smaller than that of the corresponding tokamak, but it is indeed proportional to $R_{0} a_{0}^{2}\langle\kappa\rangle$ approximately.

\section{BOOTSTRAP CURRENT AT FINITE PLASMA BETA}

In quasisymmetric stellarators, bootstrap current[20] is important to consider, as in axisymmetric tokamaks, since the physics of bootstrap current is the same in the Boozer 
TABLE II: Data of major physical quantities for different elongation cases after optimization

\begin{tabular}{|c|c|c|c|c|c|c|}
\hline$\kappa$ & Aspect ratio & $R_{\text {major }}(\mathrm{m})$ & $a_{\text {minor }}(\mathrm{m})$ & volume $\left(\mathrm{m}^{3}\right)$ & $\langle B\rangle(\mathrm{T})$ & $B_{0}(\mathrm{~T})$ \\
\hline 3.64 & 5.952 & 9.270 & 1.557 & 443.8 & 6.059 & 5.819 \\
\hline 3.50 & 5.942 & 9.258 & 1.558 & 443.6 & 6.055 & 5.815 \\
\hline 3.33 & 5.960 & 9.277 & 1.557 & 443.8 & 6.052 & 5.805 \\
\hline 3.16 & 5.977 & 9.295 & 1.555 & 443.7 & 6.052 & 5.800 \\
\hline 3.00 & 5.948 & 9.271 & 1.559 & 443.5 & 6.028 & 5.775 \\
\hline 2.94 & 5.971 & 9.287 & 1.555 & 443.5 & 6.048 & 5.793 \\
\hline 2.86 & 5.955 & 9.284 & 1.559 & 445.4 & 6.032 & 5.770 \\
\hline 2.79 & 5.953 & 9.266 & 1.557 & 443.2 & 6.052 & 5.819 \\
\hline 2.64 & 5.956 & 9.268 & 1.556 & 443.1 & 6.050 & 5.825 \\
\hline 2.57 & 5.941 & 9.257 & 1.558 & 443.7 & 6.034 & 5.829 \\
\hline
\end{tabular}

coordinates. It can significantly modify equilibrium properties through change in rotational transform. Here we use the drift-kinetic code SFINCS[21] to calculate bootstrap current. The SFINCS code solves the steady-state drift-kinetic equation for multiple species, allowing arbitrary collisionality and magnetic geometry (subject to the assumption that magnetic surfaces exist), and using the full linearized Fokker-Planck-Landau collision operator. For calculations shown here, variation of the electrostatic potential on magnetic surfaces is neglected. The SFINCS code is state of art for calculation of bootstrap current, however it is relatively expensive computationally and cannot be used directly in the optimization process. Thus here we use it iteratively in our optimization. Specifically we first calculate the bootstrap current at the start of the optimization and then keep it fixed during the optimization process using STELLOPT. After each optimization, we calculate bootstrap again using the optimized configuration and then repeat the optimization process using the new bootstrap current profile. This iterative process is repeated until the final configuration converges. We will show that this iterative method works for the cases considered in this work.

In our bootstrap current calculation, the pressure profile is given by $P_{0}\left(1-s^{2}\right)^{3}$, where $P_{0}$ is a constant adjusted by STELLOPT to achieve the target value of volume averaged beta, and $s$ is the normalized toroidal flux. The ion and electron density profiles are uniform at $4 \times 10^{20} \mathrm{~m}^{-3}$, and the temperature profiles of the two species have the same shape as the pressure profile. Fig.7 plots the current profile and total bootstrap current calculated by 


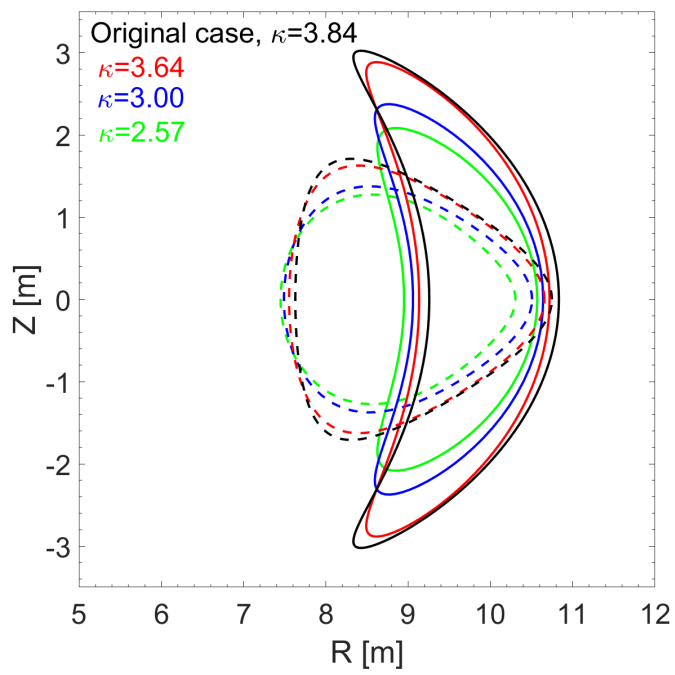

FIG. 4: Boundary flux surface variation when decreasing elongation targeting volume proportional to $\kappa$

SFINCS for different $\beta$ values for the lowest elongation $\kappa=2.57$ case in Fig.2. We observe that the dependence of the bootstrap current profile on the $\beta$ value and pressure profile are consistent with theoretical expectation [22]). It should be noted that the central iota decreases as beta increases. This is due to the effect of beta on equilibrium.

Fig. 8 shows the total bootstrap current as a function of $\kappa$, holding the plasma $\beta=0.05$. The curve for the constant volume series indicates that the total bootstrap current increases a little when the elongation decreases. The other curve shows that the bootstrap current is approximately proportional to elongation or volume. In tokamaks, the bootstrap current density is given by $\langle J \cdot B\rangle=-c R B_{\phi} d P / d \psi$ or $\langle J \cdot B\rangle=-c\left(B_{\phi} / B_{p}\right) d P / d r$ where $\mathrm{c}$ is a constant coefficient, $\psi$ is poloidal flux, $B_{p}$ is poloidal magnetic field[22]. Thus the elongation affects total bootstrap current through the cross section area and the poloidal field. The poloidal B field in stellarator is mainly supplied by external coils when bootstrap current is small. In this limit, the bootstrap current is proportional to elongation since cross section area or plasma volume is proportional to elongation. This explains why in FIG.8, the total bootstrap current is roughly independent of kappa for the constant volume case, while for the varying volume case, the total bootstrap current is almost proportional to kappa (volume). 


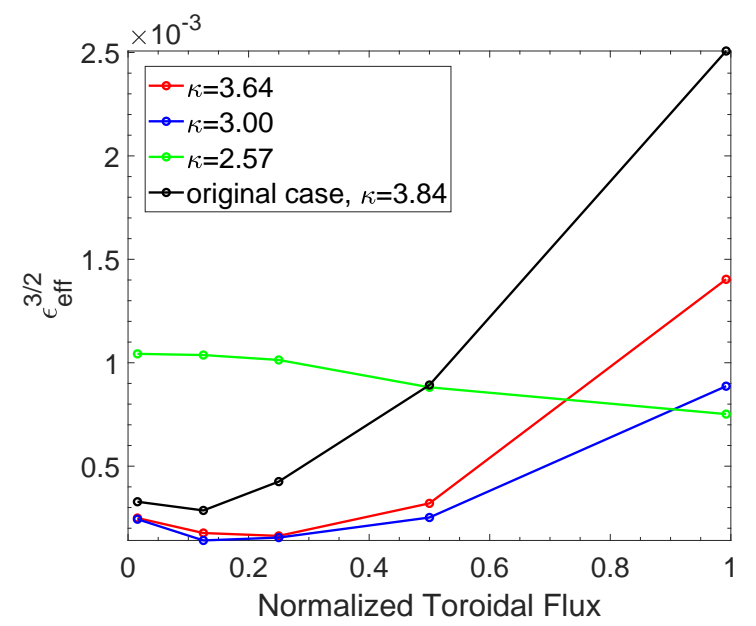

FIG. 5: Effective ripple variation when decreasing elongation targeting volume proportional to $\kappa$

\section{MHD STABILITY OPTIMIZATION}

MHD stability is an important property for all magnetic confinement devices. Strong global MHD instabilities can seriously degrade the confinement of plasmas. Therefore it is important to consider MHD stability in designing optimized stellarators.

We first consider the stability of MHD modes for the $\kappa=2.57$ case with plasma beta value of $\beta=0.05$. The equilibrium profiles of pressure, current, and rotational transform are shown in Fig.9.

We see that the rotational transform profile is very different from the zero beta case (see Fig. 1(c)). Iota increases radially from about 0.1 at the magnetic axis to 0.75 at $s=0.75$ and then decreases to 0.6 at the edge. Fig. 10 shows the 5 dominant Fourier harmonics for the $n=0$ and $n=1$ families calculated by the TERPSICHORE code[23, 24]. We note that, for the $n=0$ mode family, the largest component $((\mathrm{m}, \mathrm{n})=(4,3))$ peaks near the $\iota=0.75$ rational surface as expected. On the other hand, for the $n=1$ mode family, the mode is clearly external with the dominant $(2,1)$ component peaks at the edge. This is consistent with the fact that iota $=0.5$ is the the nearest major rational surface in the vacuum. The normalized eigenvalues for the two families $(n=0$ and $n=1)$ are $\omega^{2}=-2.269 \times 10^{-2}$ and $-4.988 \times 10^{-2}$ respectively. The magnitudes of the eigenvalue indicate that the modes are strongly unstable. Thus these modes need to be stabilized via optimization.

As done above we keep the bootstrap current profile fixed as given in Fig.9(b) although the 


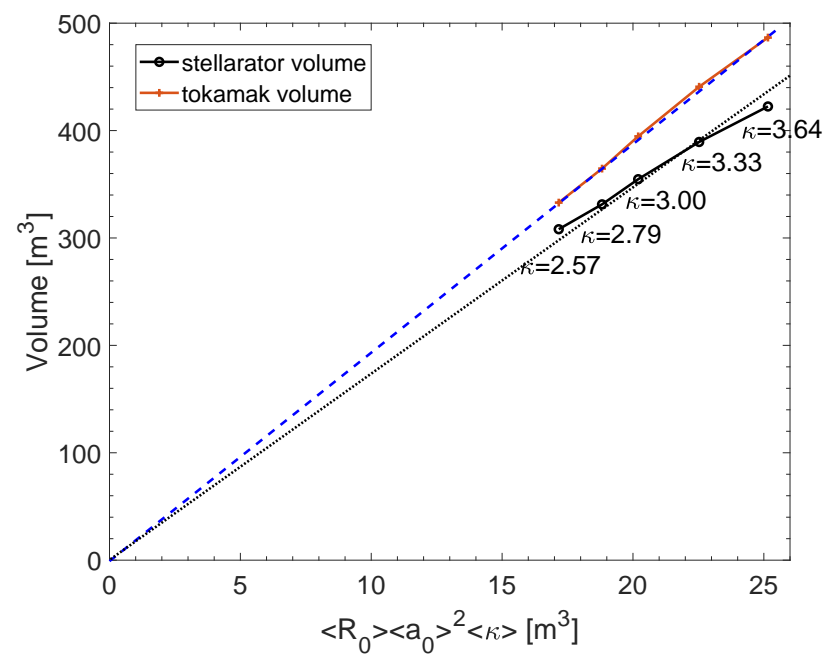

FIG. 6: Volume versus $R_{0} a_{0}^{2}\langle\kappa\rangle$

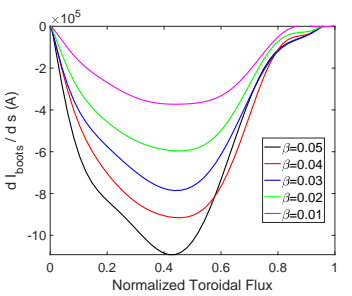

(a)Current density profile, where

$I_{\text {boots }}$ is the enclosed toroidal

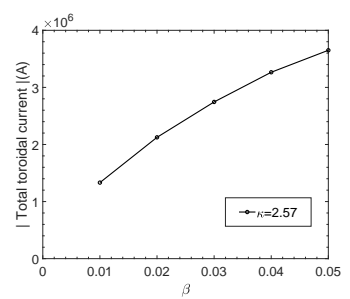

(b)Total bootstrap current

magnitude versus plasma $\beta$

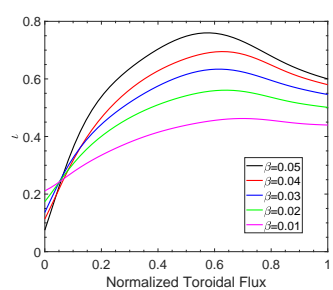

(c)Rotational transform profile

for different plasma $\beta$ cases

current in a normalized flux s

FIG. 7: Bootstrap current for different $\beta$ in the $\kappa=2.57$ case plotted in Fig.2

plasma boundary will be changed as the optimization progresses due to the computational expense of calculating the bootstrap current by SFINCS. We use a Levenberg-Marquardt (L-M) algorithm[25, 26] with finite differences for derivative evaluation in our optimization when targeting MHD stability. The total number of function evaluations required to achieve a satisfactory optimization is significantly less for L-M than for GADE, and trapping in local minima was not found to be a significant problem. 


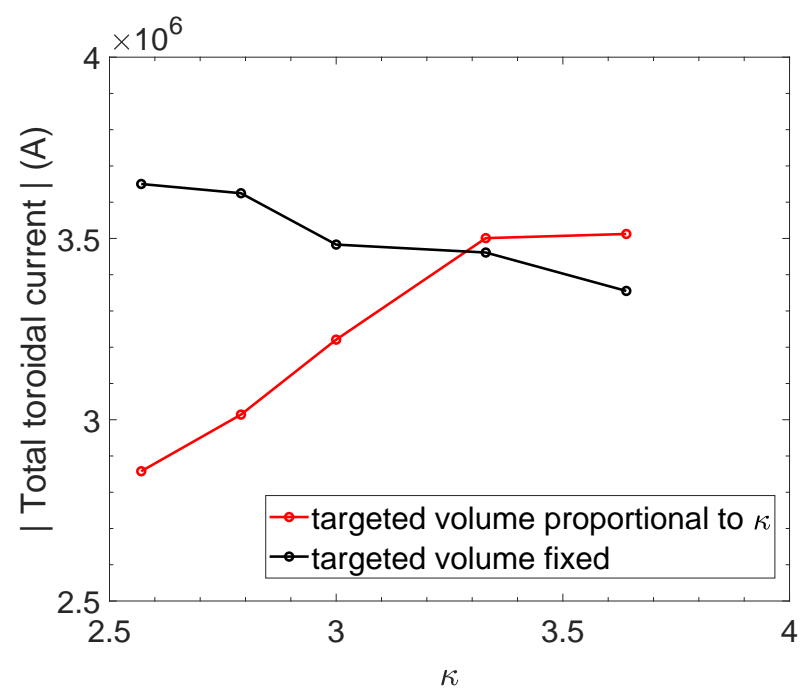

FIG. 8: Bootstrap current vs $\kappa$ for the two series of elongation scan with $\beta=0.05$

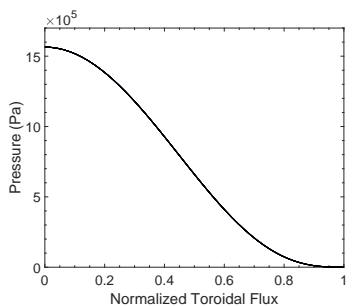

(a)Pressure profile

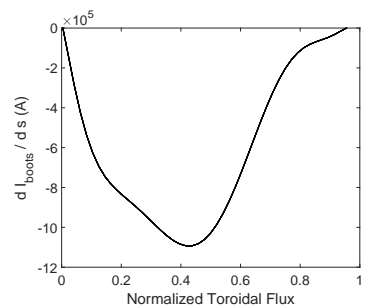

(b)Bootstrap current profile

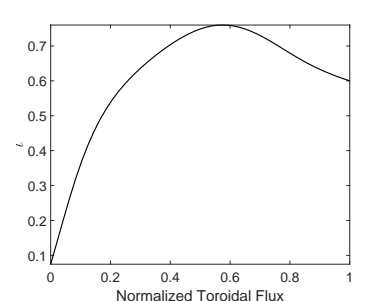

(c)Rotational transform profile

FIG. 9: Pressure, current, and rotational transform profiles for $\beta=0.05$ in the $\kappa=2.57$ case plotted in Fig.2

Fig.11(a) shows the evolution of key targets including kink stability in the STELLOPT calculation for the above case. We observe that the unstable kink growth rate squared value is reduced over 4 orders of magnitude, but the neoclassical transport level is increased somewhat. The later iterations are mainly optimizing the neoclassical transport. Fig.11(d) shows clearly that the negative curvature at the inner side of the flux surface at $\phi=0$ plane is increased, i.e., higher triangularity. This is consistent with our expectation that the negative curvature improves the MHD stability[27]. Fig.12 shows that it requires larger 


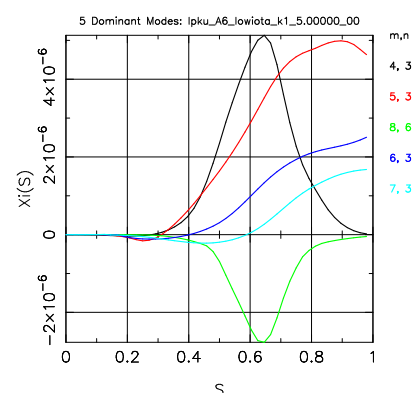

(a) $n=0$ family

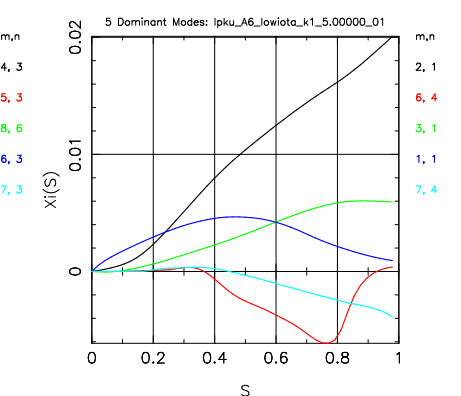

(b) $\mathrm{n}=1$ family

FIG. 10: 5 dominant mode structures for $n=0$ and $n=1$ families calculated by TERPSICHORE, where $\mathrm{Xi}$ is the normalized amplitude of plasma displacement

negative curvature for the higher plasma $\beta$ cases.

Since the flux surfaces are changed after optimization, we need to re-calculate the bootstrap current again to make it consistent with the new configuration, and check the MHD stability again. If it is kink unstable, we need to do the optimization again. The iterative process needs to be repeated a few times until the results are converged. Fig.13 shows evolution of key targets during the iteration. Using the final case of Fig.11, we calculate its self-consistent bootstrap current $j_{b s 1}(\beta=0.05)$ and find that it is kink unstable. After the 2 nd optimization, the final case with its self-consistent bootstrap current $j_{b s 2}$ is much more stable $\left(\omega^{2}=1.29 \times 10^{-5}\right.$ and $1.64 \times 10^{-5}$ for $n=0$ and $n=1$ family respectively), and the neoclassical transport level is also relatively low (Fig.13(b)).

\section{CONCLUSION}

In this work a systematic optimization study has been carried out starting from a 3 field periods quasi-symmetric stellarator with aspect ratio of 6 . A series of zero beta QA configurations with varied elongation are generated using the stellarator optimization code STELLOPT. For finite beta stellarator plasmas the bootstrap current is calculated selfconsistently using SFINCS code. The bootstrap current increases approximately linearly with elongation for QA stellarators, if the volume is proportional to elongation. Good QA stellarators are obtained with varied plasma beta, self-consistent bootstrap current, low neoclassical transport and good kink stability. This study demonstrates that good QA stellarators with varied elongation and plasma beta exist. Future work will explore larger parameter space of quality QA stellarator configurations. 


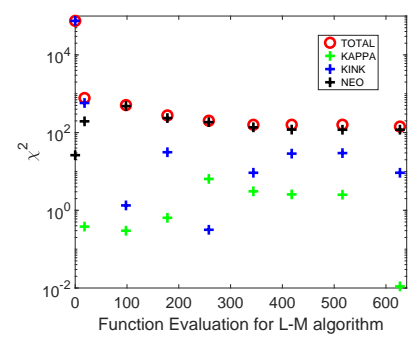

(a) $\chi^{2}$ evolution

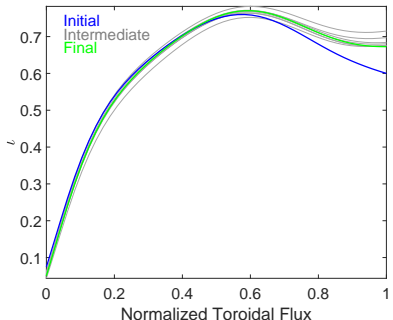

(c) $\iota$ evolution

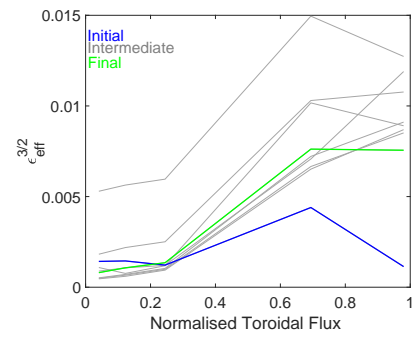

(b)Effective ripple evolution

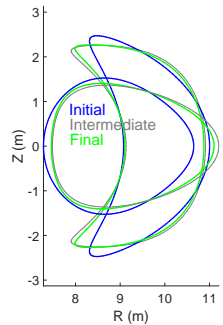

(d)Boundary flux shape evolution at

$$
\phi=0 \text { and } \phi=\pi / \mathrm{nfp}
$$

FIG. 11: Progress of kink optimization using STELLOPT with Levenberg-Marquardt algorithm with finite difference derivative evaluation

\section{Acknowledgments}

This work is supported by Zhejiang Universitys startup funding for one of the authors (Guoyong Fu) and by the US Department of Energy under Contract Number DE-AC02$09 \mathrm{CH} 11466$.

[1] Boozer A.H., 1981 Plasma equilibrium with rational magnetic surfaces, Phys. Fluids 24 19992003

[2] J. Nührenberg, and R.Zille, 1988 Quasi-helically symmetric toroidal stellarators, Physics Letters A 129, Issue 2, 113-117

[3] Canik J.M., Anderson D.T., Anderson F.S.B., Likin K.M., Talmadge J.N. and Zhai K., 2007 


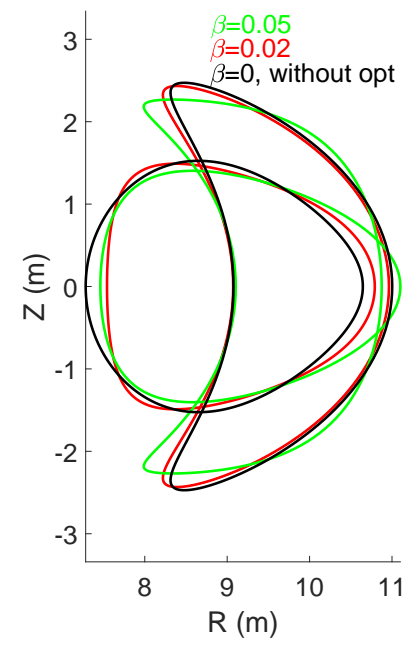

FIG. 12: Boundary flux surfaces after kink optimization for different plasma $\beta$

Experimental demonstration of improved neoclassical transport with quasihelical symmetry, Phys. Rev. Lett. 98085002

[4] Nührenberg J., Lotz W. and Gori S., 1994 Quasi-axisymmetric tokamaks Theory of Fusion Plasmas, Varenna, 1994(Bologna: Editrice Compositori) p 3;

[5] Garabedian P.R., 1996 Stellarators with the magnetic symmetry of a tokamak, Phys. Plasmas 32483

[6] B.E. Nelson, L.A. Berry, A.B. Brooks, M.J. Cole, J.C. Chrzanowski, H.-M. Fan, P.J. Fogarty, P.L. Goranson, P.J. Heitzenroeder, S.P. Hirshman et al, 2003 Design of the national compact stellarator experiment (NCSX), Fusion Eng. Des. 66 169-174

[7] S. Okamura, K. Matsuoka, S. Nishimura, M. Isobe, I. Nomura, C. Suzuki, A. Shimizu, S. Murakami, N. Nakajima, M. Yokoyama et al, 2001 Physics and engineering design of the low aspect ratio quasi-axisymmetric stellarator CHS-qa, Nucl. Fusion 411865

[8] Drevlak M., Brochard F., Helander P., Kisslinger J., Mikhailov M., Nührenberg C., Nührenberg J. and Turkin Y., 2013 ESTELL: a quasi-toroidally symmetric stellarator Contrib, Plasma Phys. 53 459-468 


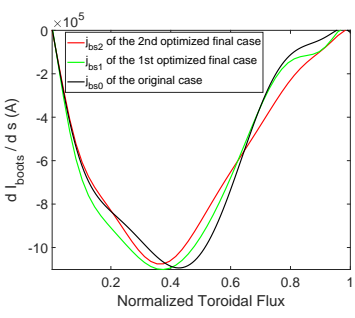

(a)Bootstrap current profile

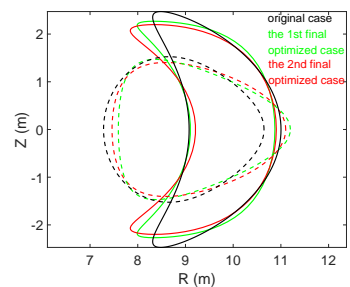

(c)Boundary flux surfaces

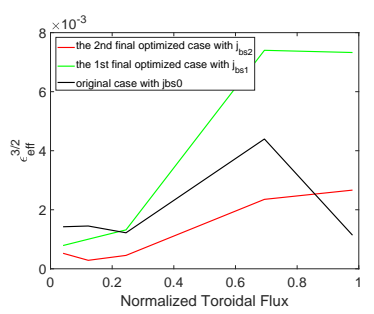

(b)Effective ripple variation

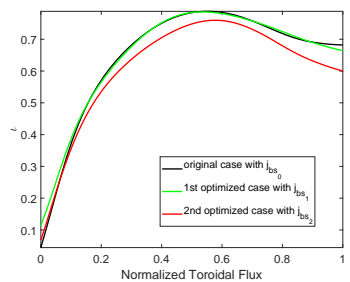

(d)Rotational transform variation variation

FIG. 13: Self consistent bootstrap current profiles of the optimized results, the effective ripple, the corresponding boundary flux surfaces, and the rotational transform profiles

[9] Y. H. Xu, private communication, 2019

[10] S.A. Henneberg, M. Drevlak, C. Nührenberg, C.D. Beidler, Y. Turkin, J. Loizu and P. Helander, 2019 Properties of a new quasi-axisymmetric configuration, Nucl. Fusion 59026014

[11] L. P. Ku,P. R. Garabedian,J. Lyon,A. Turnbull,A. Grossman,T. K. Mau,M. Zarnstorff and ARIES Team, 2008 Physics Design for ARIES-CS, FUSION SCIENCE AND TECHNOLOGY 54,673

[12] D.A. Gates, A.H. Boozer, T. Brown, J. Breslau, D. Curreli, M. Landreman, S.A. Lazerson, J. Lore, H. Mynick, G.H. Neilson et al, 2017 Recent advances in stellarator optimization, Nucl. Fusion 57126064

[13] A. M. M. Todd, J. Manickam, M. Okabayashi, M.S. Chance, R.C. Grimm, J.M. Greene, J.L. Johnson, 1979 Dependence of ideal-MHD kink and ballooning modes on plasma shape and profiles in tokamaks, Nucl. Fusion 19, 743 
[14] J. E. Kinsey, R. E. Waltz, and J. Candy, 2007 The effect of plasma shaping on turbulent transport and $\mathrm{E} \times \mathrm{B}$ shear quenching in nonlinear gyrokinetic simulations, Phys. Plasmas 14, 102306

[15] L.P. Ku and A.H. Boozer, 2010 Modular coils and plasma configurations for quasiaxisymmetric stellarators, Nucl. Fusion 50125005

[16] S.A. Lazerson STELLOPT https://www.osti.gov/doecode/biblio/12551-stellopt

[17] Melanie Mitchell, 1996 An Introduction to Genetic Algorithms. Cambridge, MA: MIT Press.

[18] Storn R. Price K., 1997 Differential evolution - a simple and efficient heuristic for global optimization over continuous spaces. Journal of Global Optimization. 11: 341C359.

[19] V.V. Nemov and S.V. Kasilov, 1999 Evaluation of 1/n neoclassical transport in stellarators, PHYSICS OF PLASMAS, 6, 4622

[20] R.J. Bickerton J. W. Connor and J.B. Taylor, 1971 Diffusion Driven Plasma Currents and Bootstrap Tokamak, Nature Phys. Sci. 229110

[21] M. Landreman, H. M. Smith, A. Molln, and P. Helander, 2014 Comparison of particle trajectories and collision operators for collisional transport in nonaxisymmetric plasmas, Phys. Plasmas 21042503

[22] O. Sauter, C. Angioni, and Y. R. Lin-Liu, 1999 Neoclassical conductivity and bootstrap current formulas for general axisymmetric equilibria and arbitrary collisionality regime, Physics of Plasmas 6, 2834

[23] D.V Anderson, W.A. Cooper, R. Gruber, S. Merazzi and U. Schwenn, 1990 Methods for the Efficient Calculation of the (MHD) Magnetohydrodynamic Stability Properties of Magnetically Confined Fusion Plasmas, The International Journal of Supercomputing Applications, $4(3), 34 \mathrm{C} 47$.

[24] G.Y. Fu, W.A. Cooper, R. Gruber, U. Schwenn, and D.V. Anderson, 1992 Fully threedimensional ideal magnetohydrodynamic stability analysis of low-n modes and Mercier modes in stellarators, Physics of Fluids B: Plasma Physics 4, 1401

[25] Kenneth Levenberg, 1944 A Method for the Solution of Certain Non-Linear Problems in Least Squares, Quarterly of Applied Mathematics. 2: 164C168.

[26] Donald W. Marquardt, 1963 An Algorithm for Least-Squares Estimation of Nonlinear Parameters, SIAM Journal on Applied Mathematics. 11 (2): 431C441. 
[27] Okabayashi M., Asakura N., and Bell R., 1989 Initial results of the PBX-M experiment. International Atomic Energy Agency (IAEA): IAEA. 


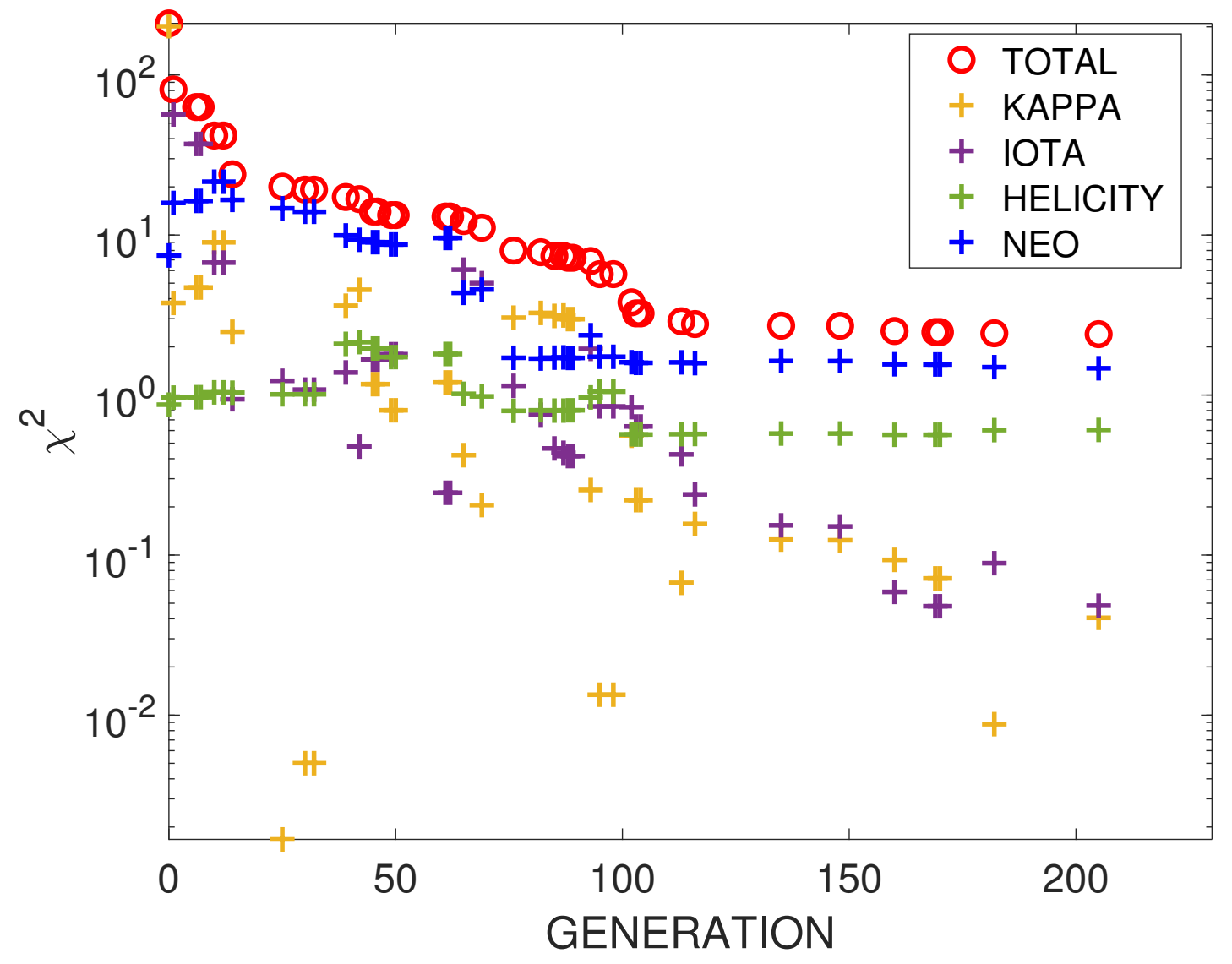




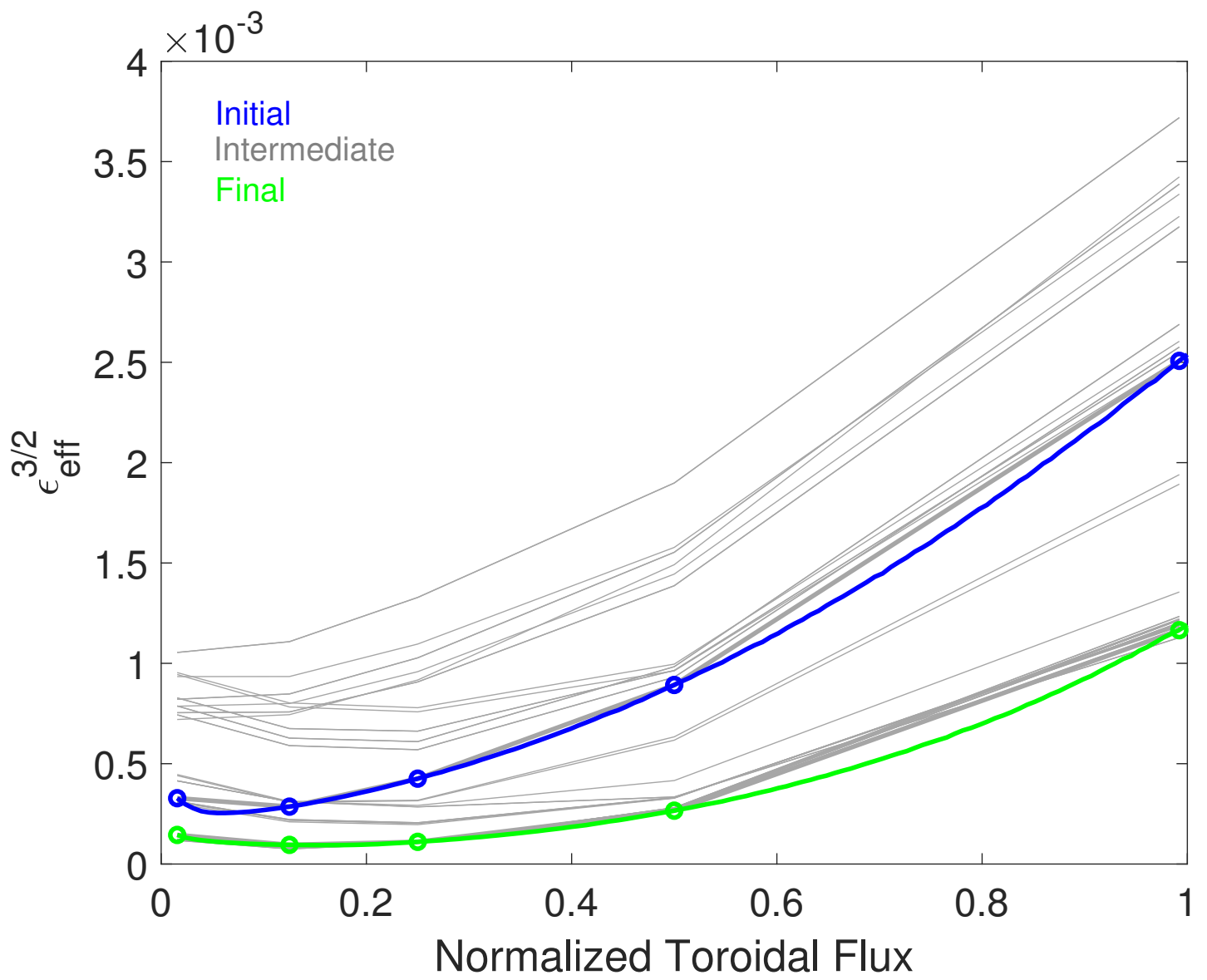




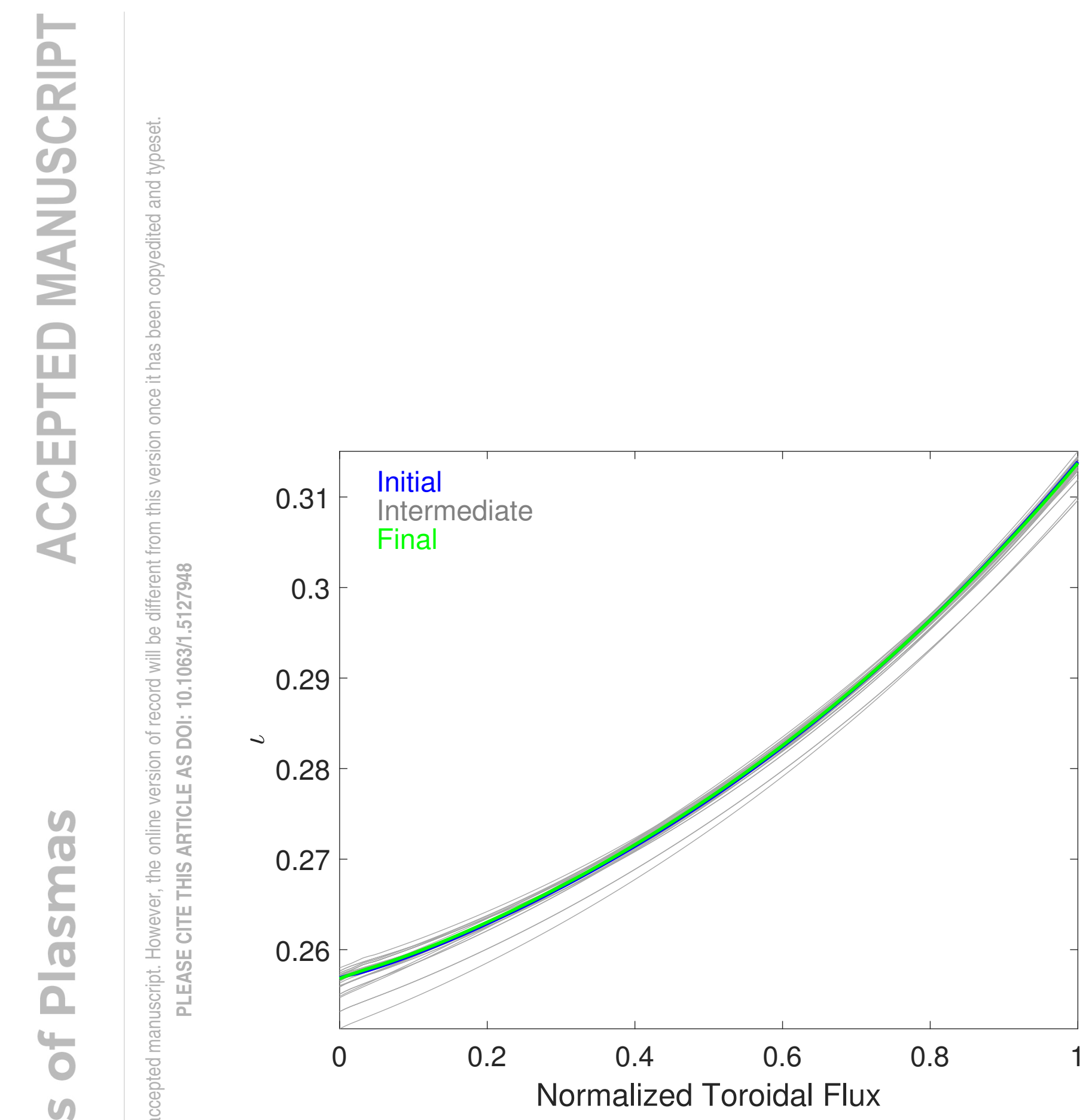



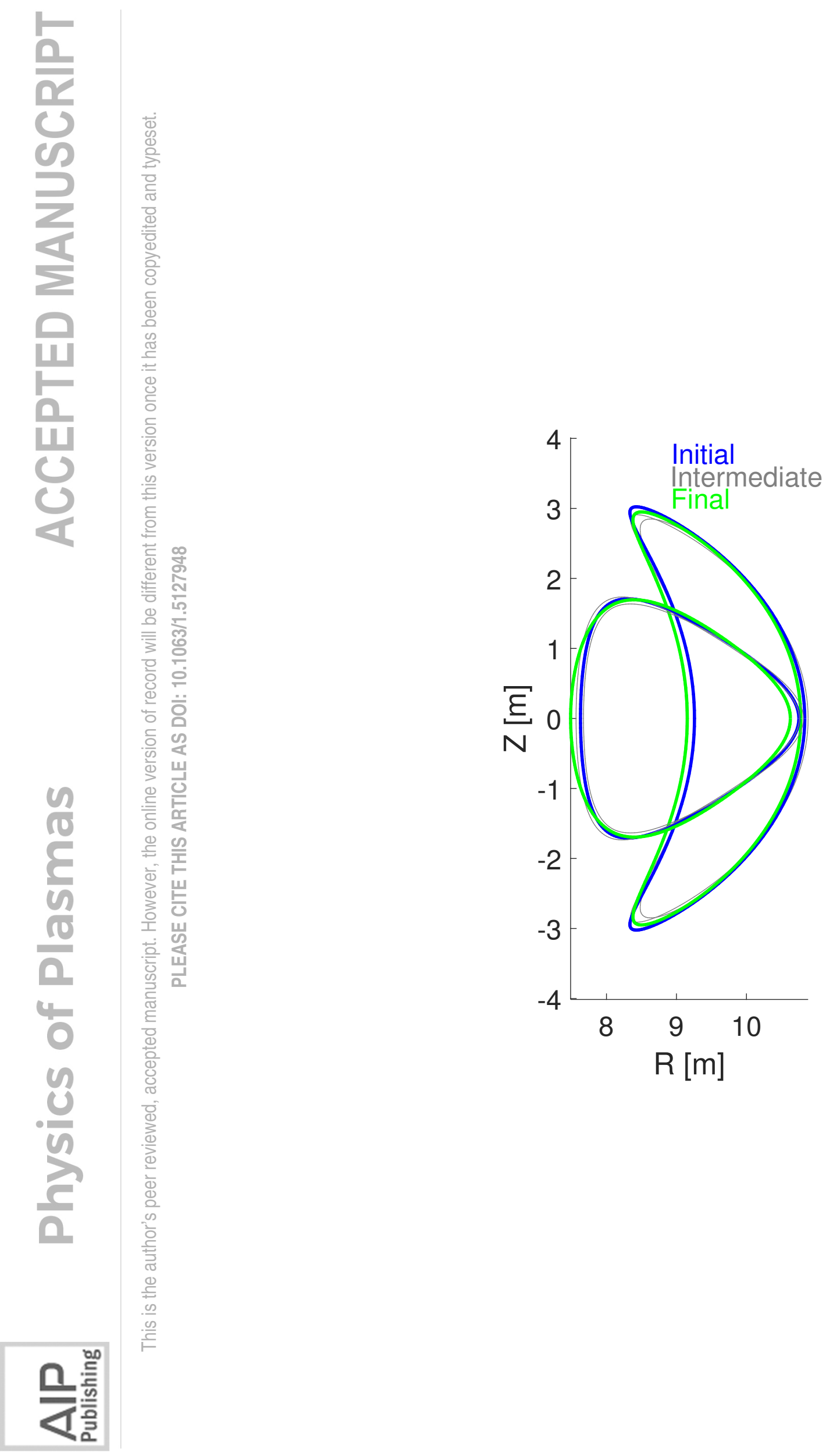


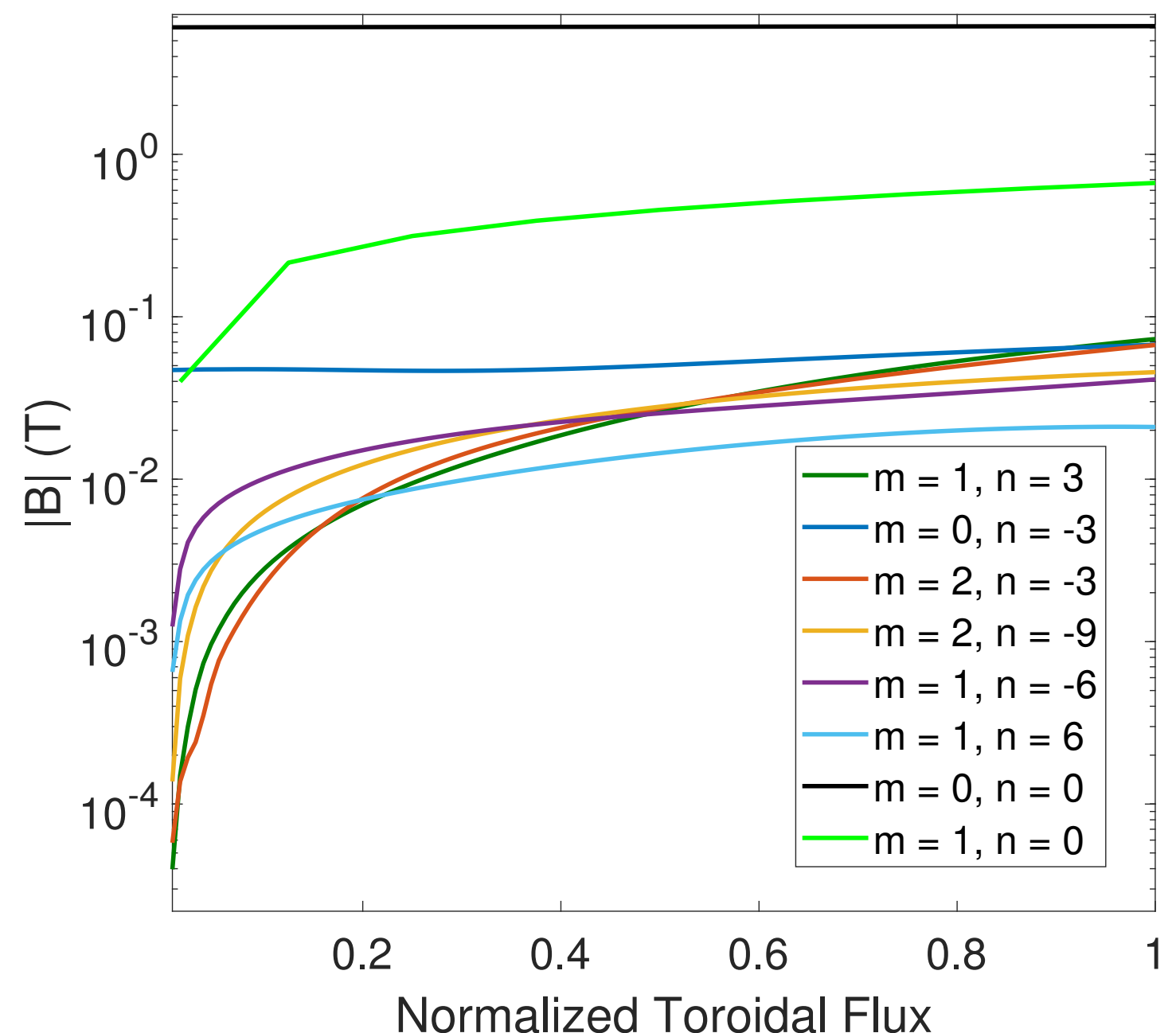




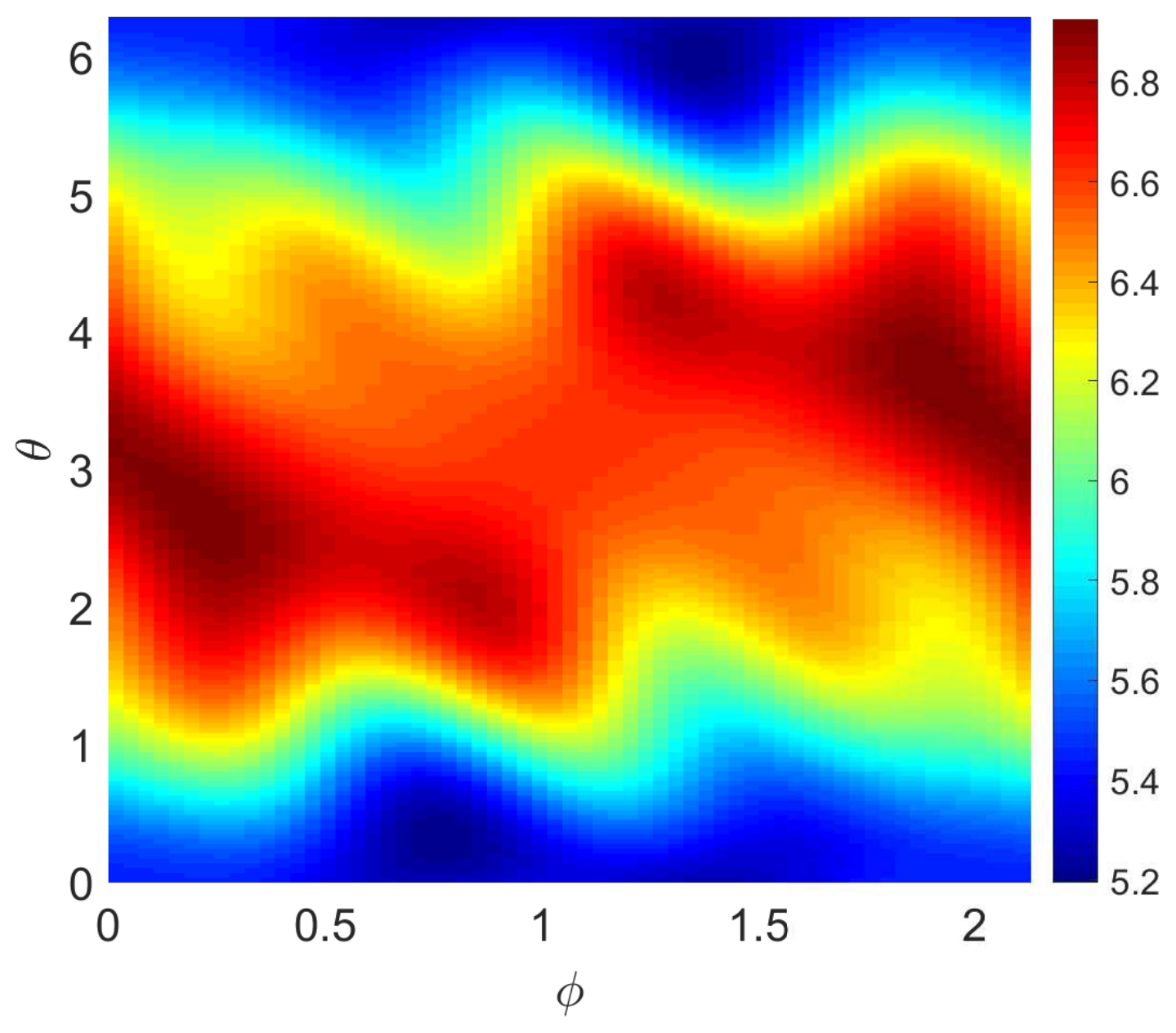




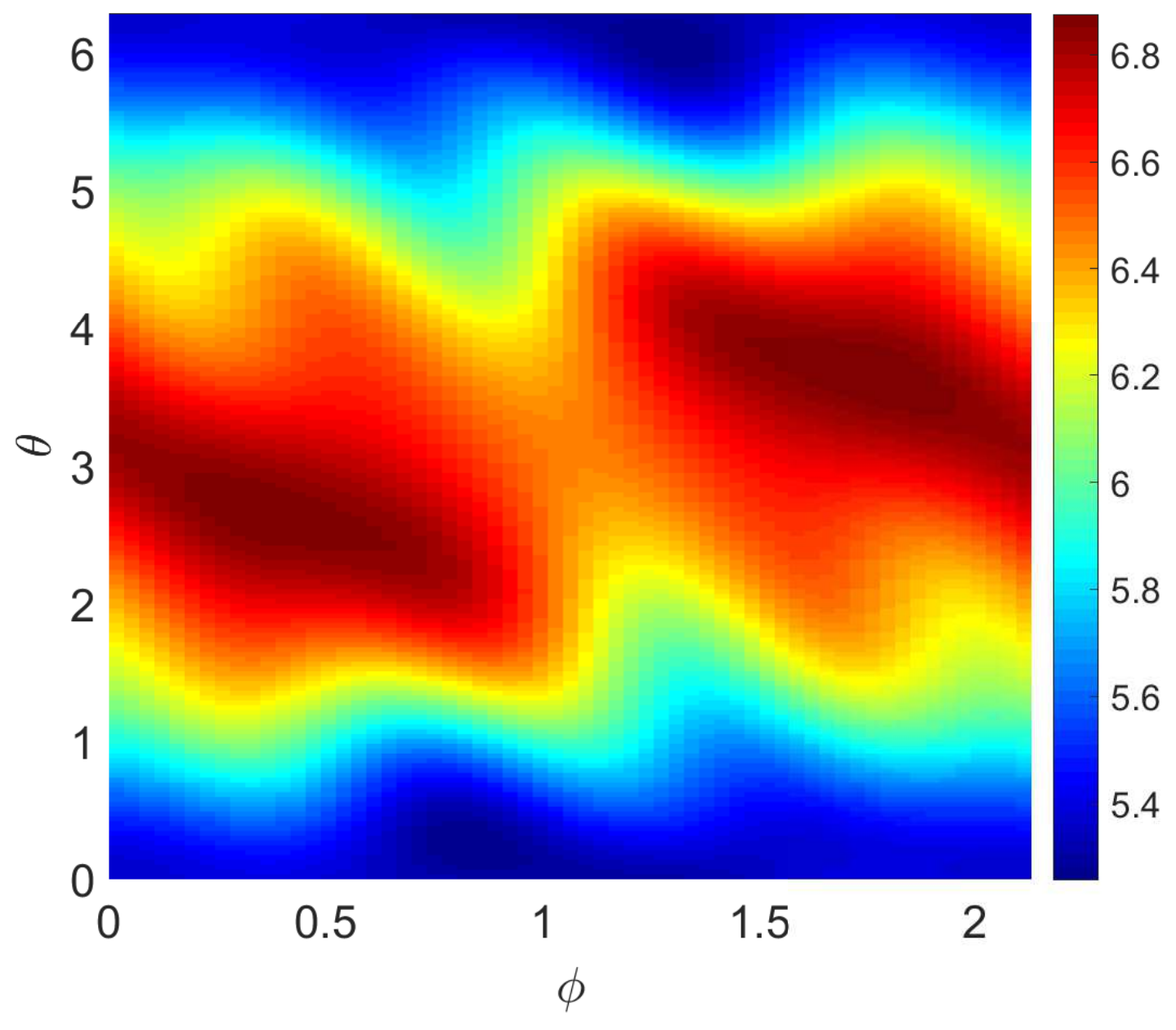




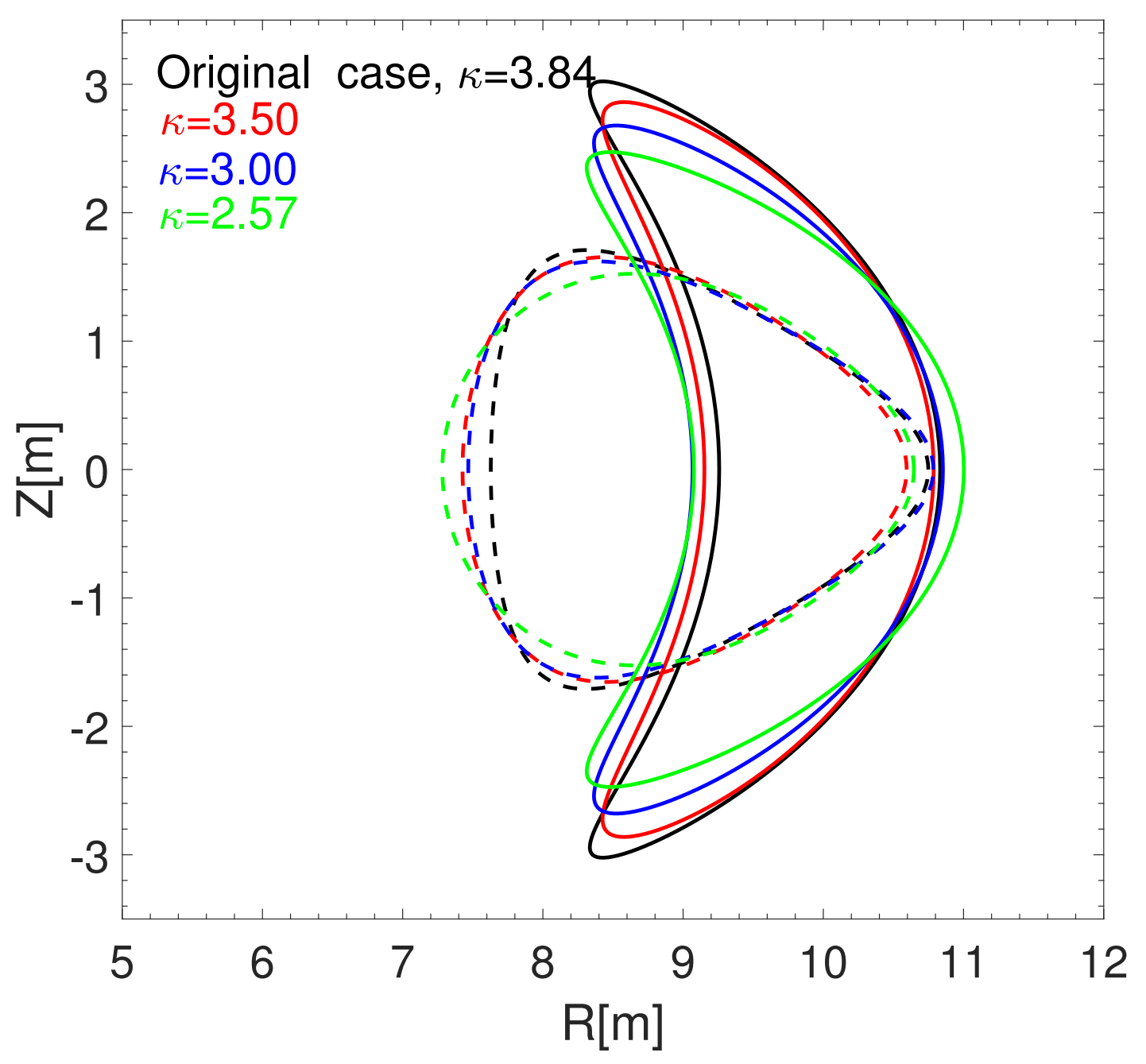

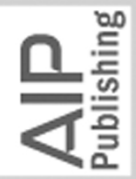




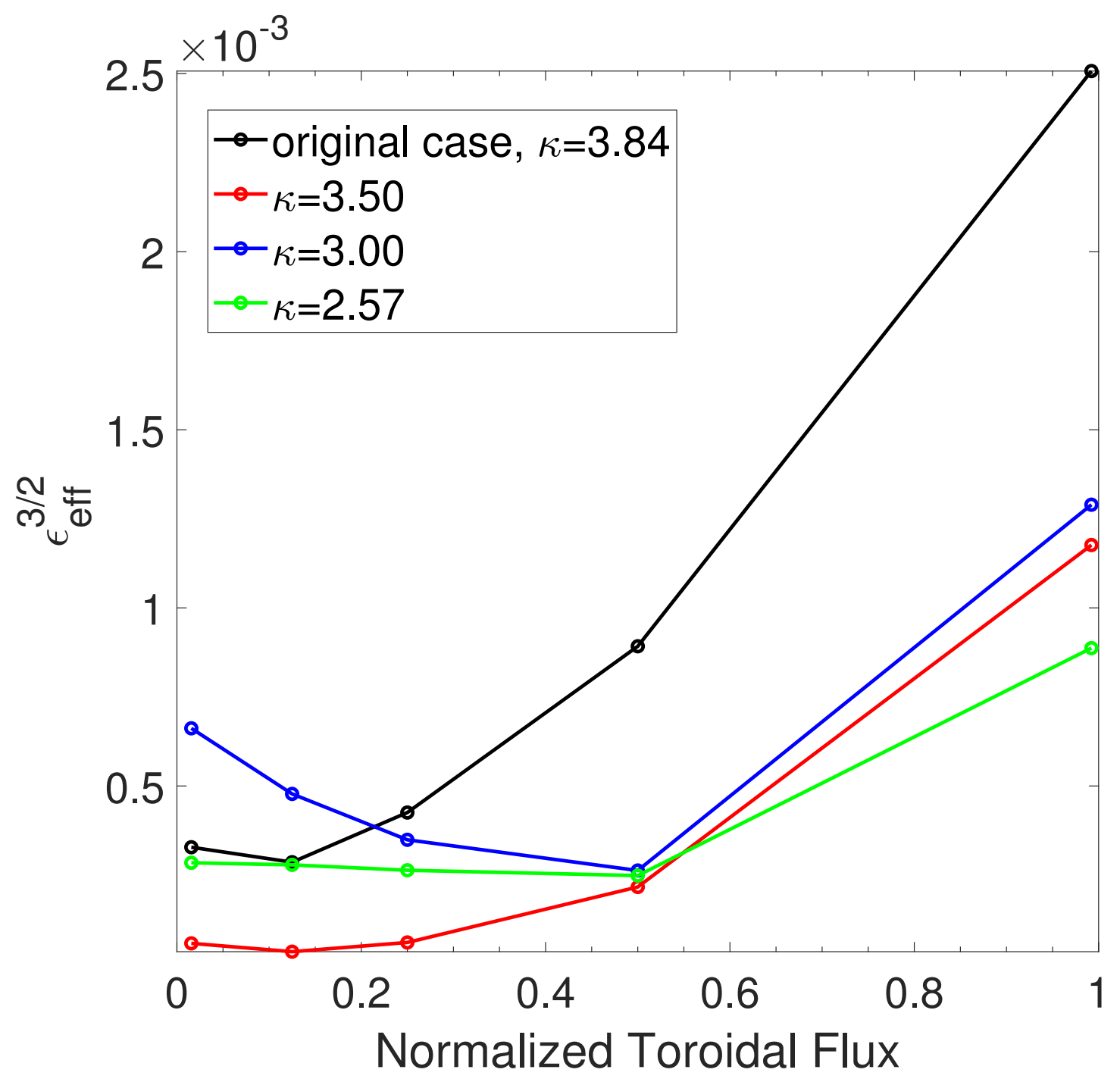




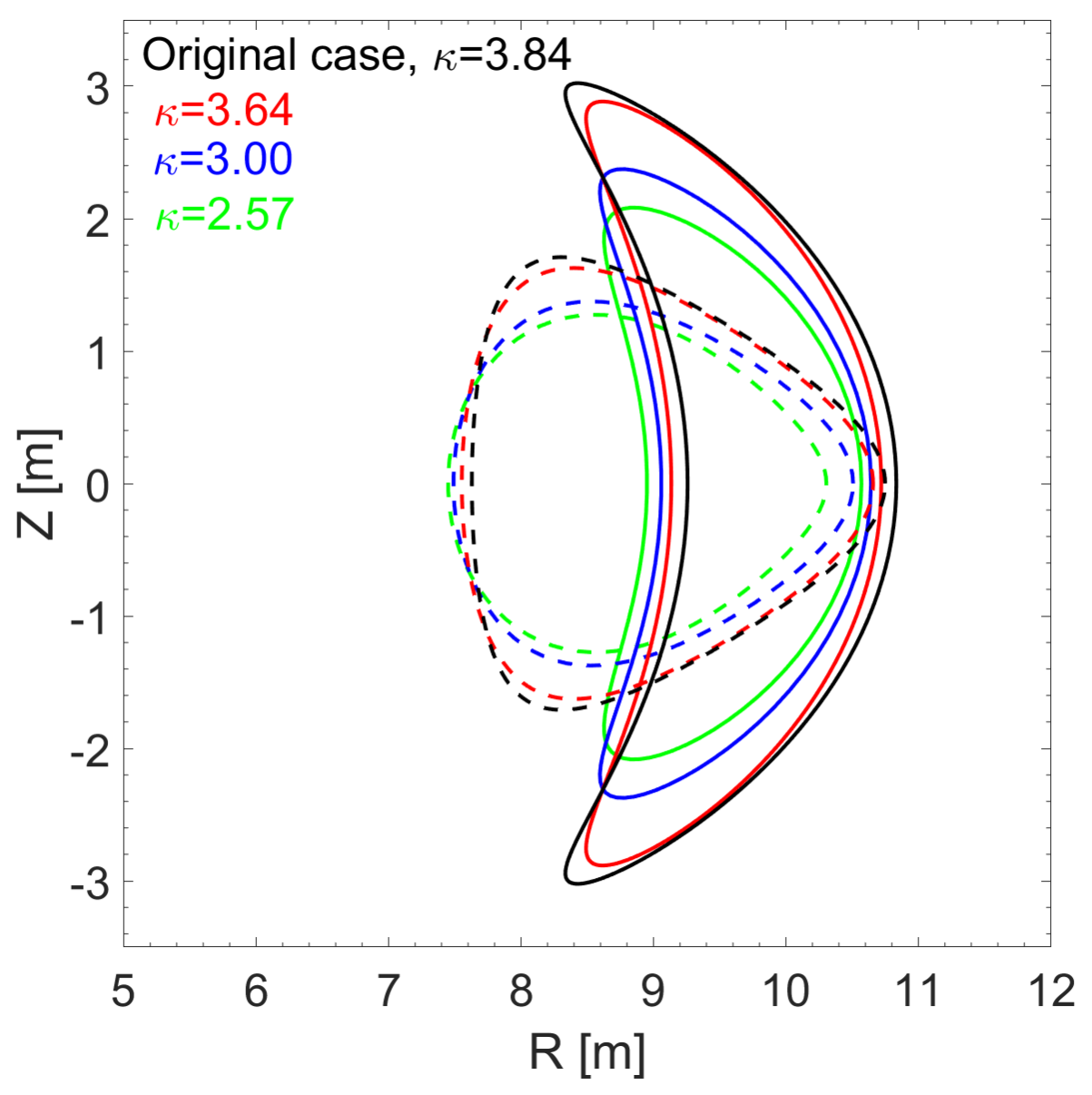

吅喜产 


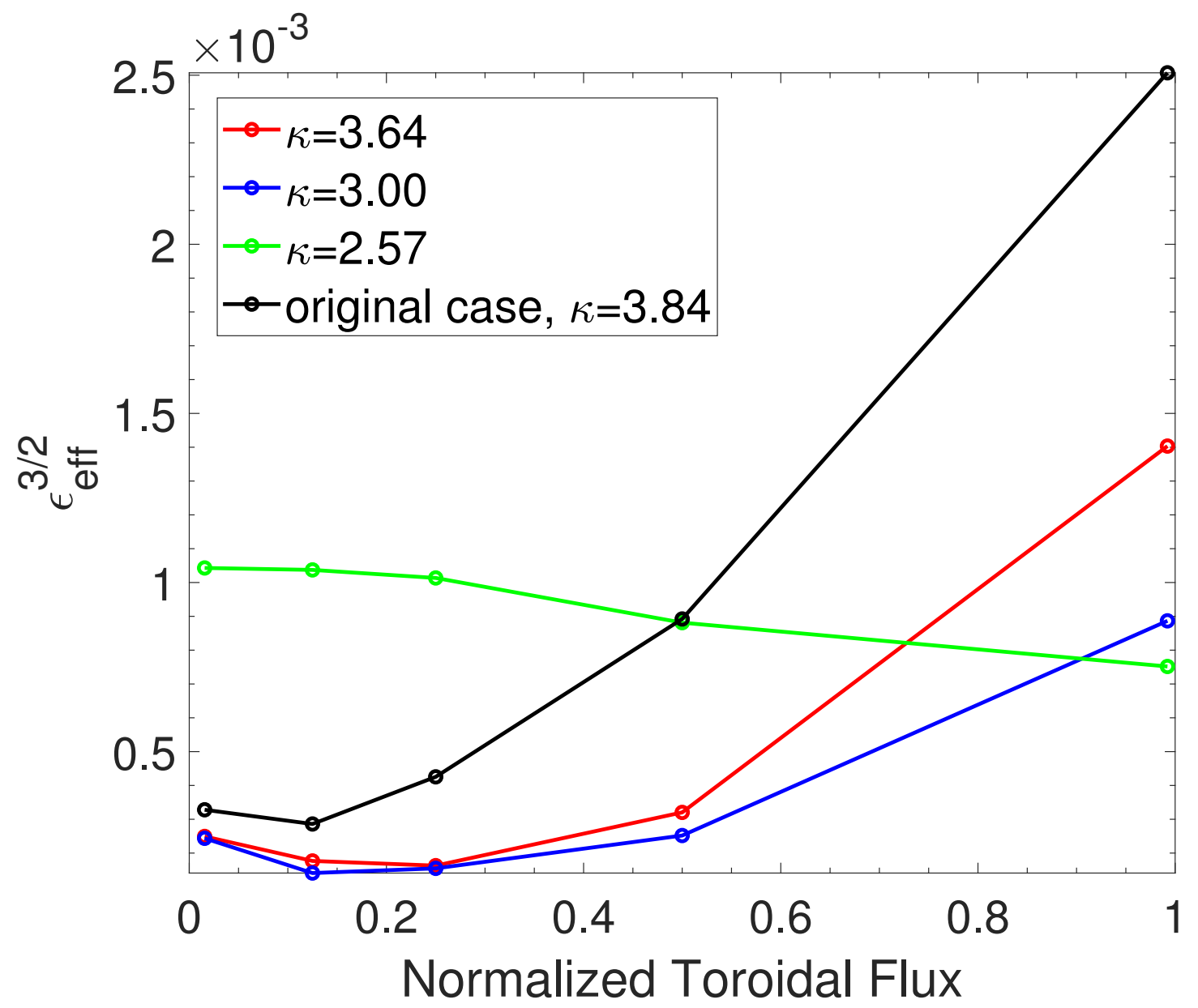




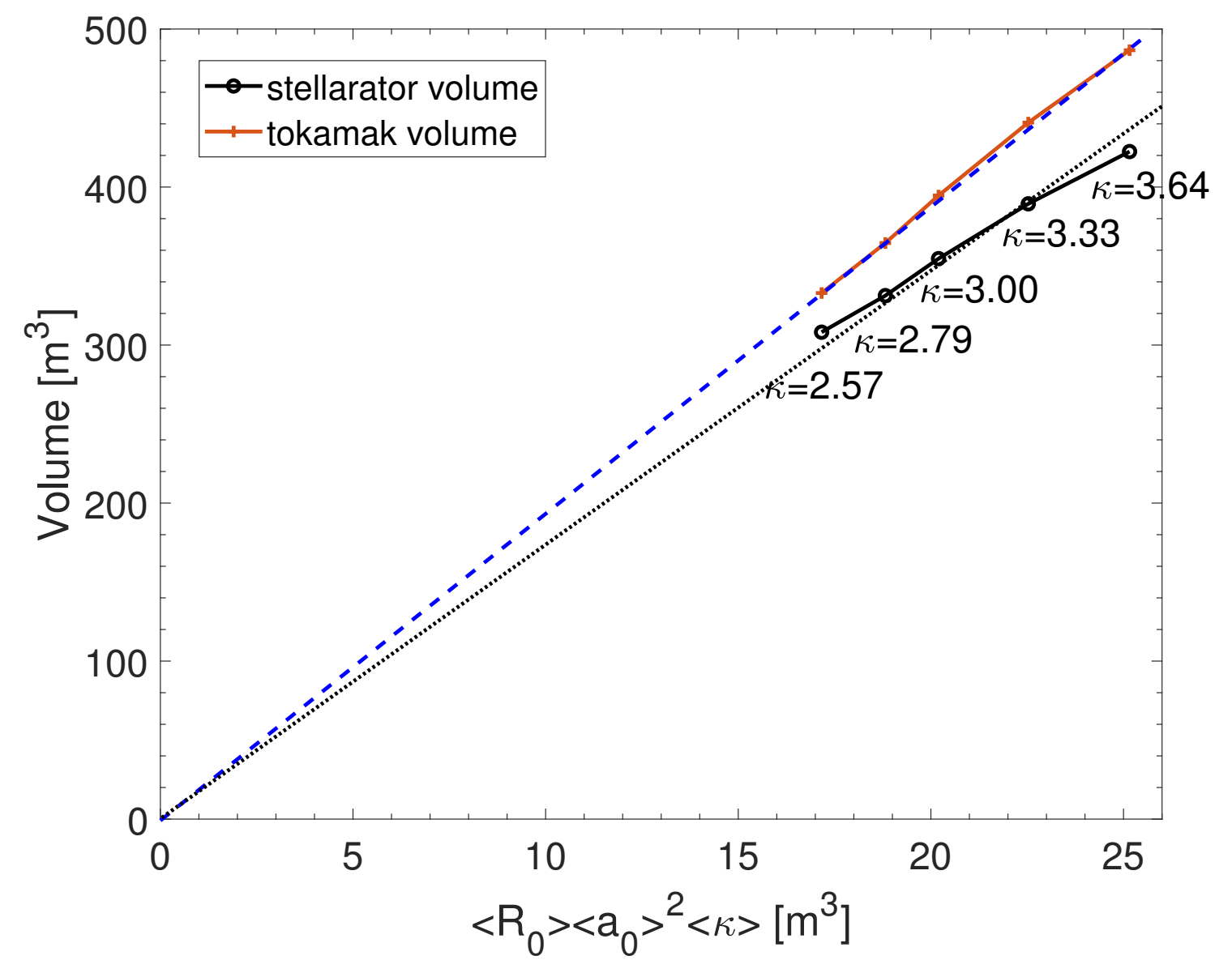




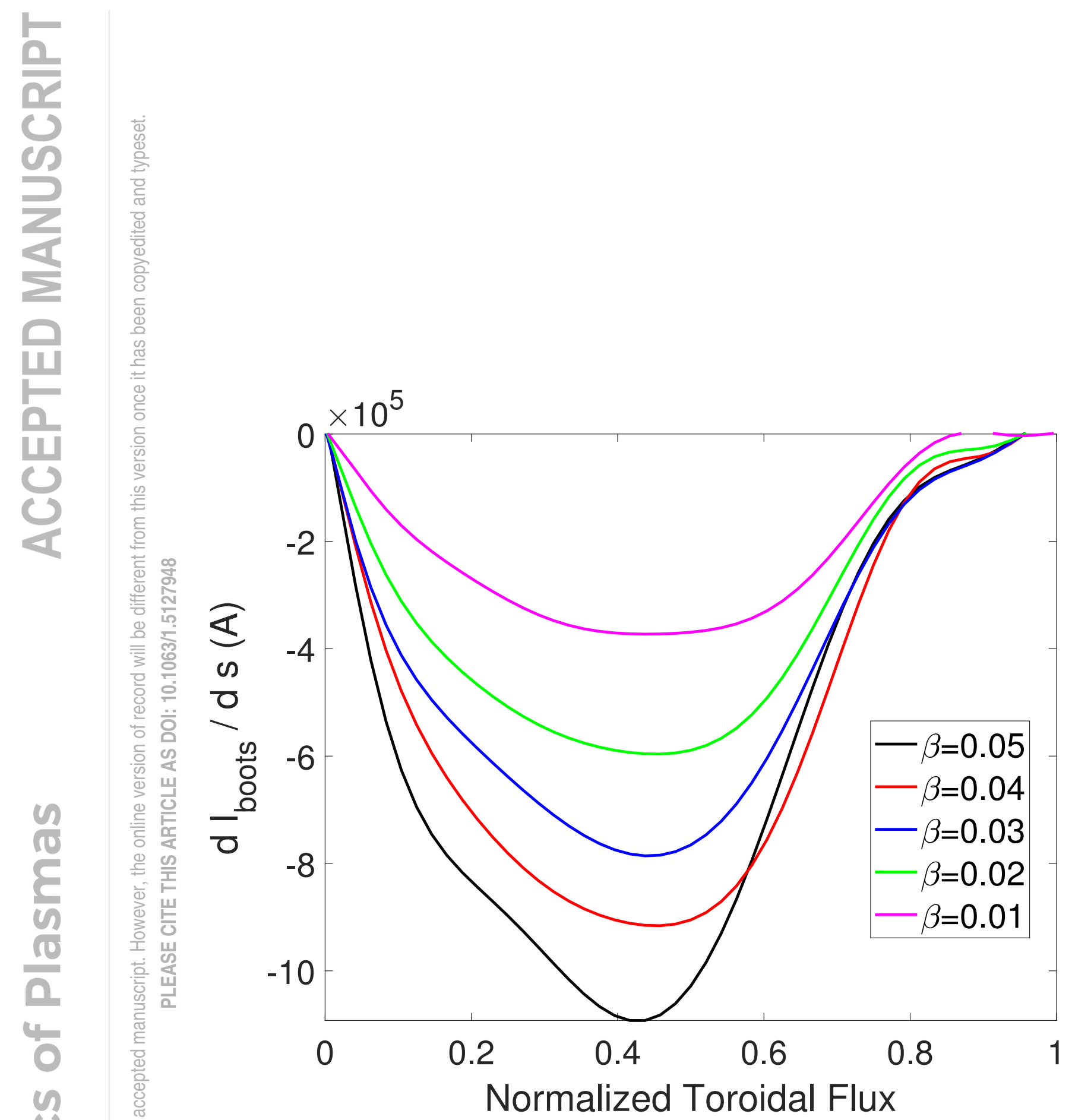

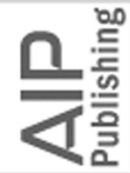




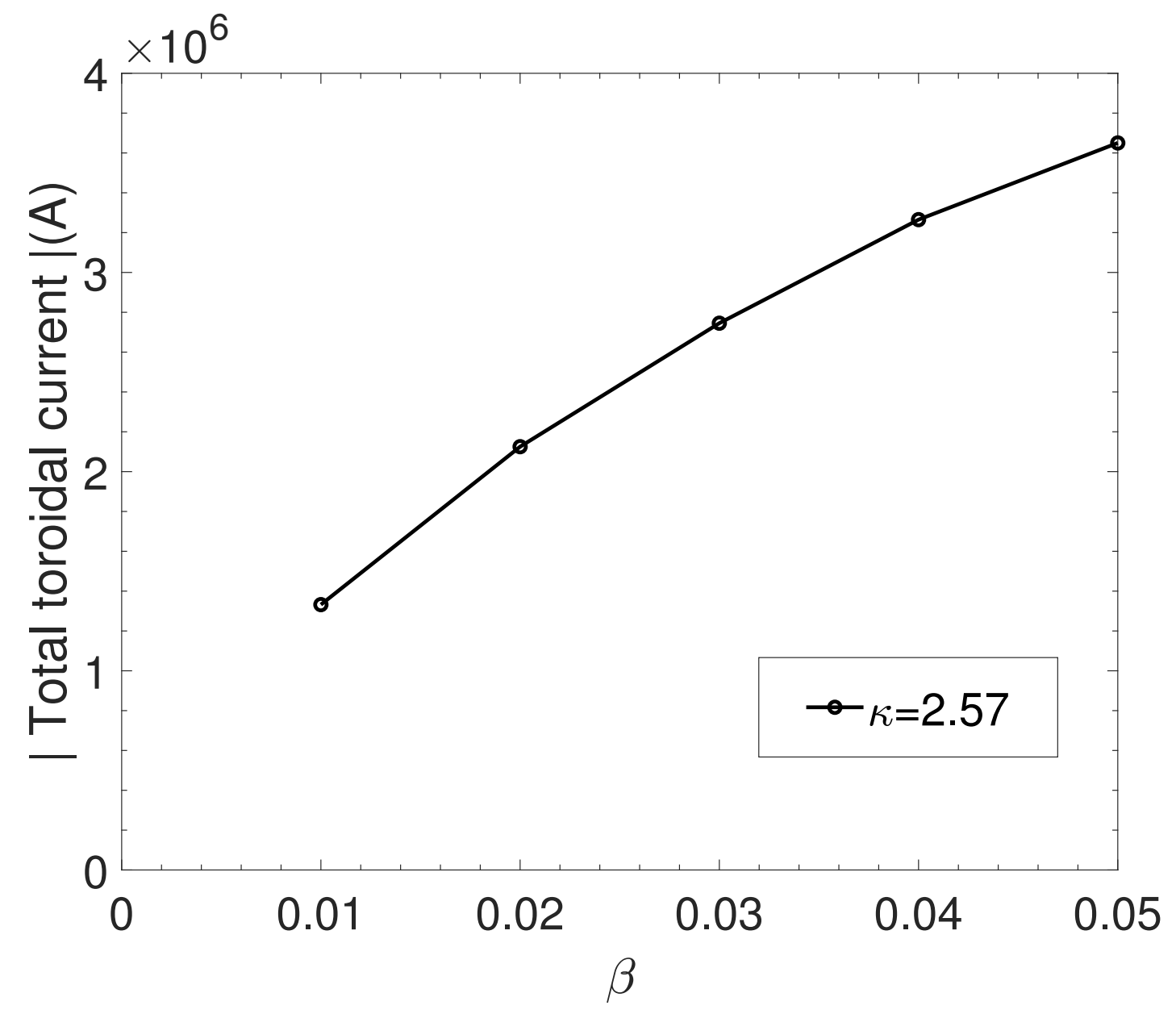

는 


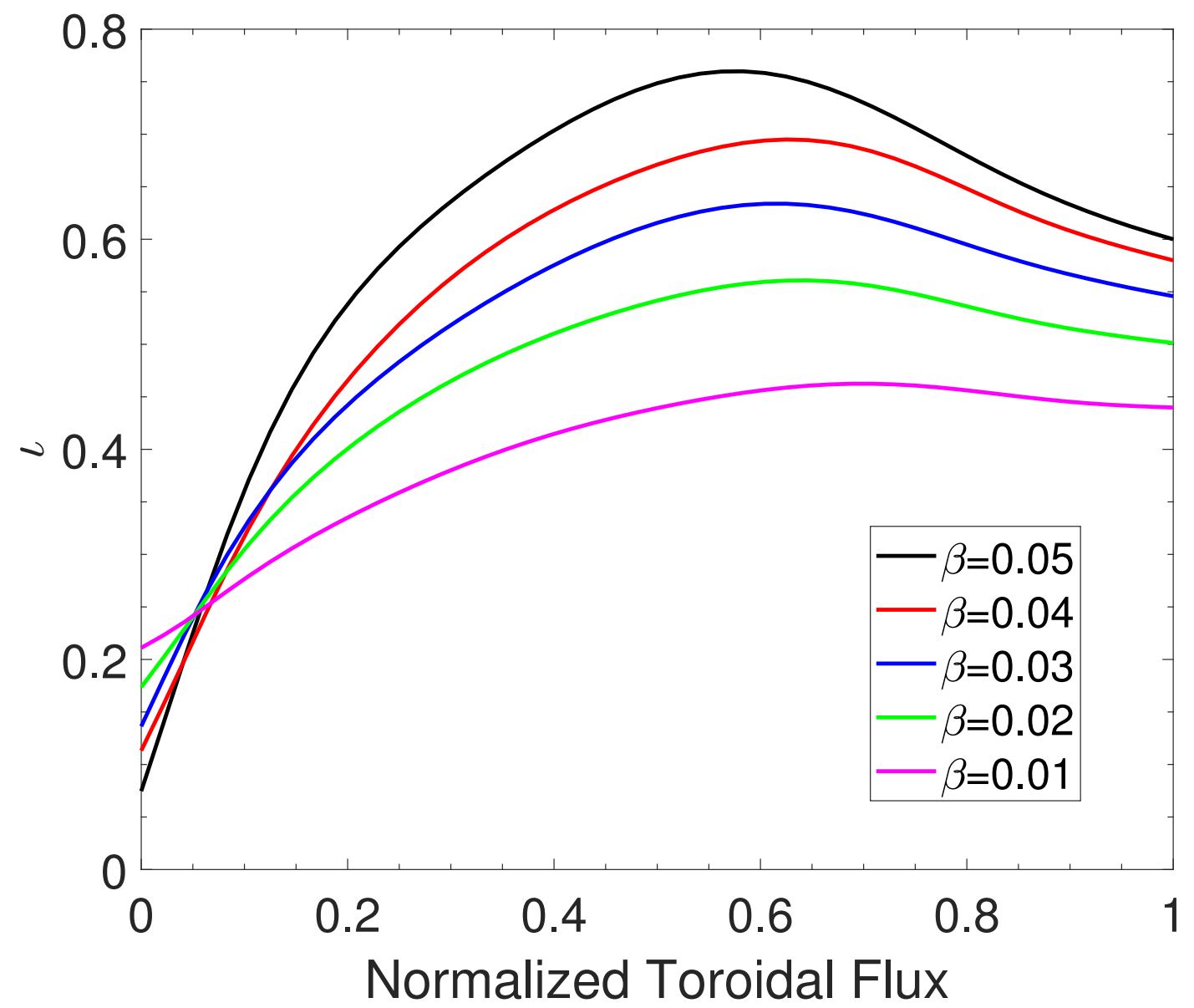

兄耪言 


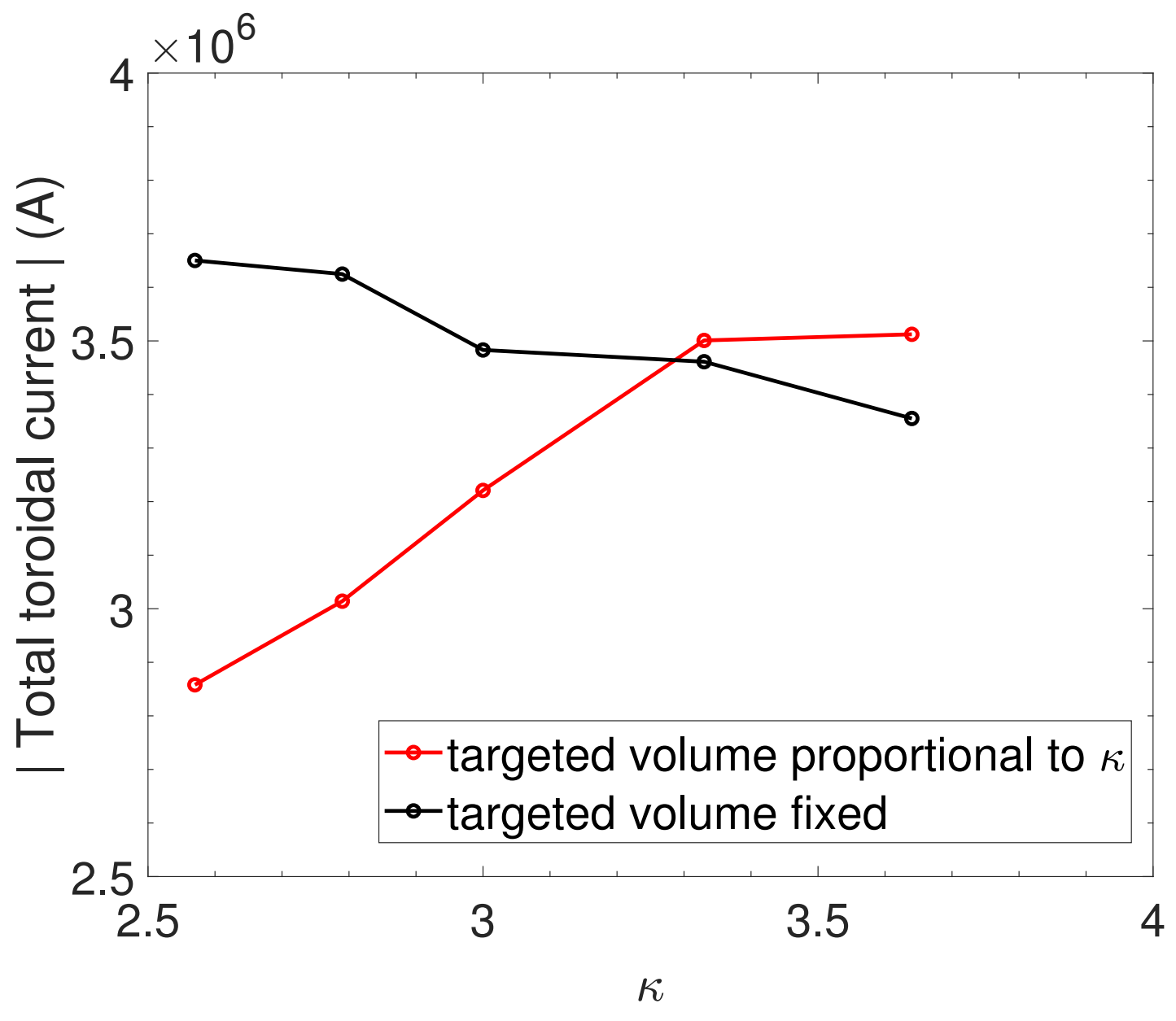




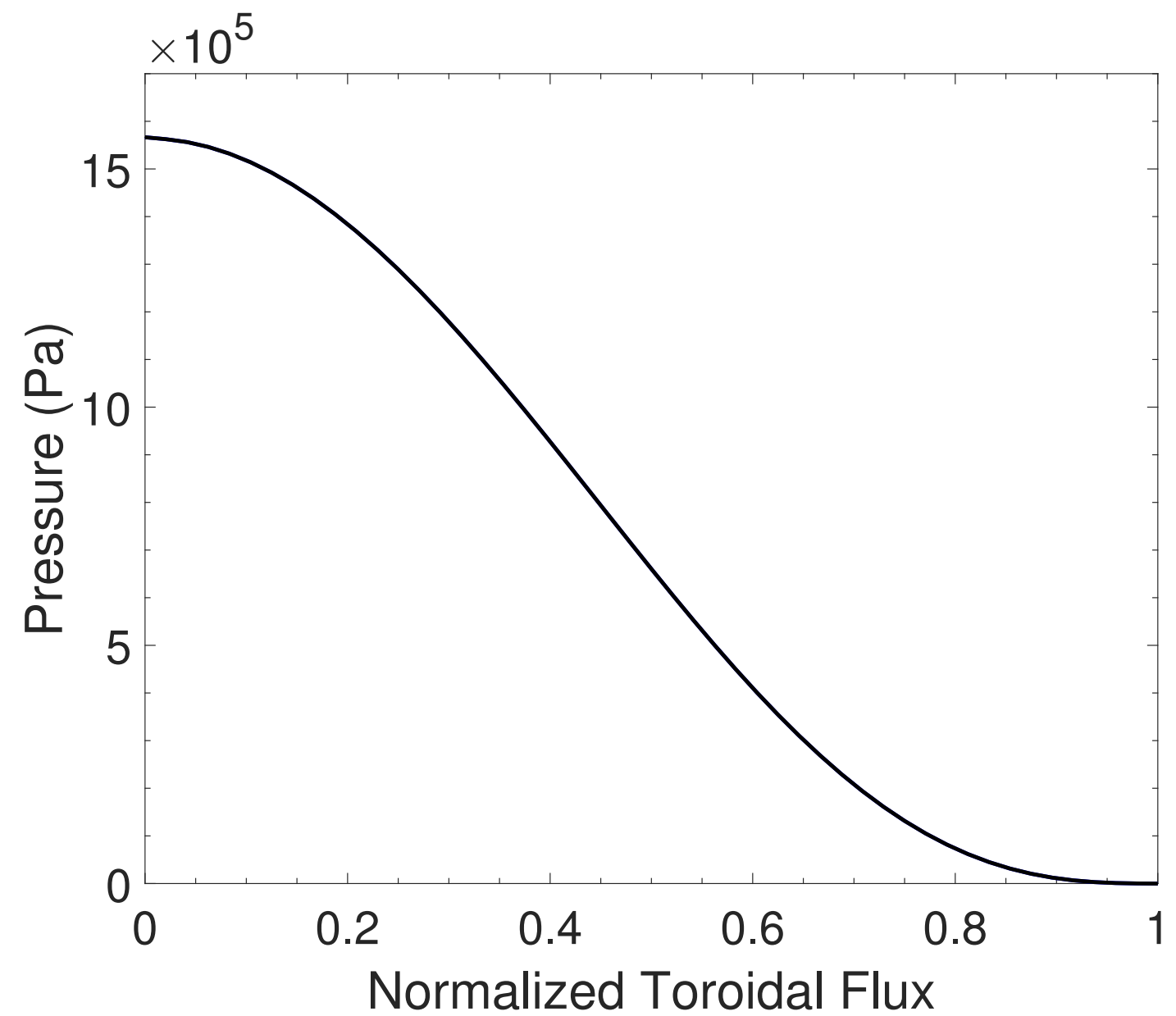

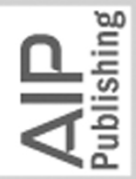




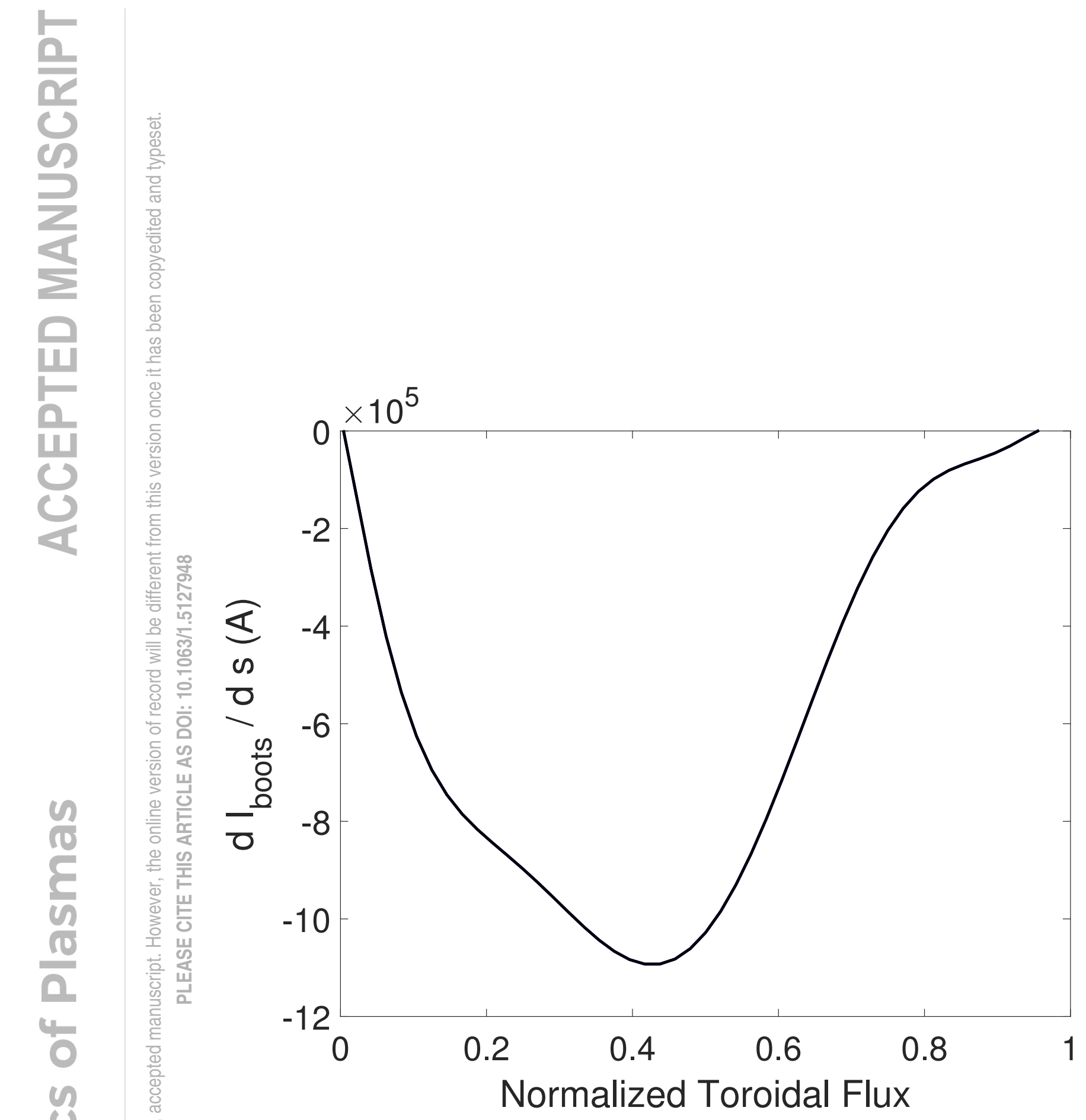

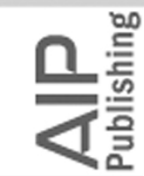




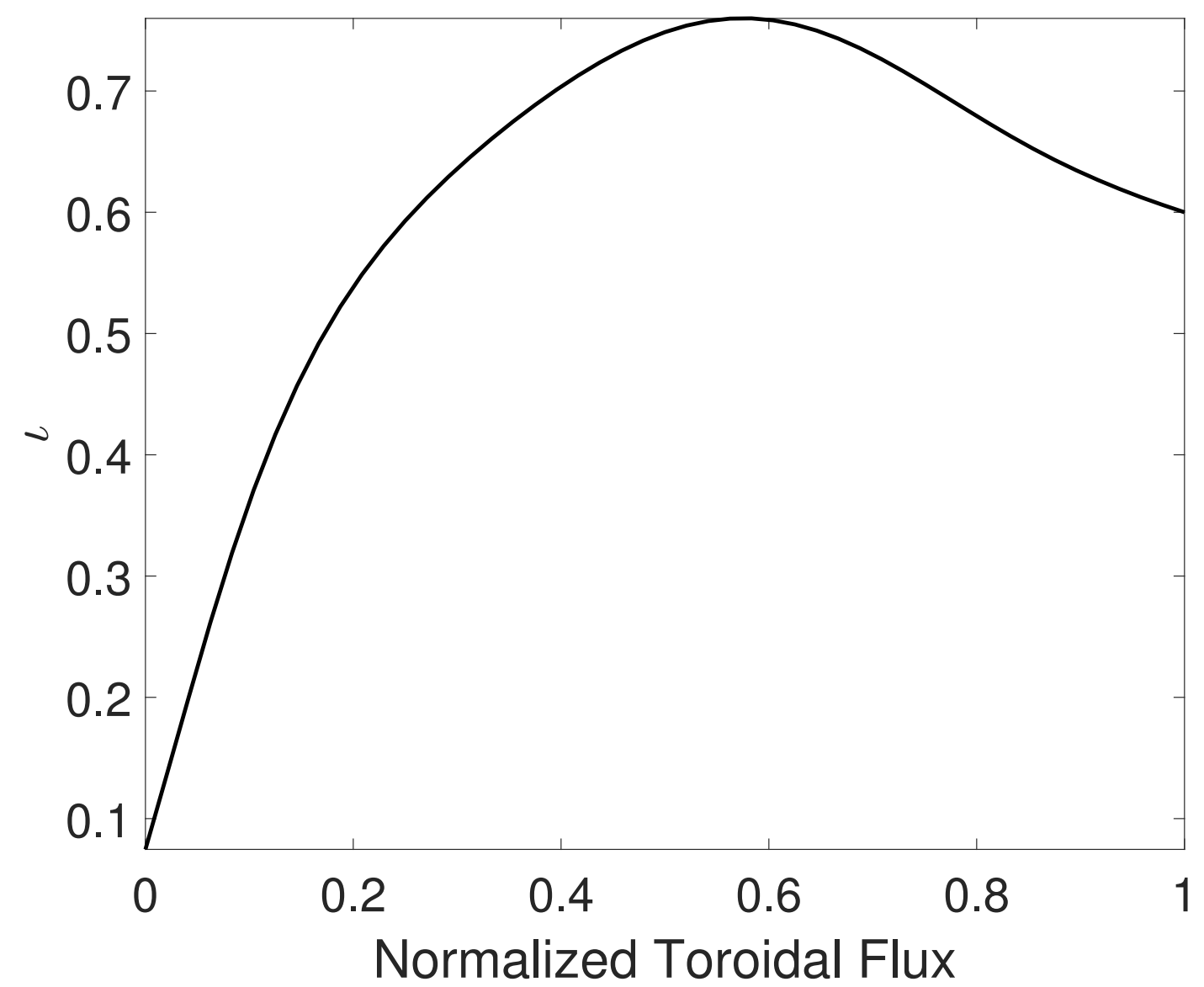

는 
$\begin{array}{llllll}E & m & m & 0 & m & m \\ E & f & 0 & 0 & 0 & \cdots\end{array}$

$\frac{1}{4}$

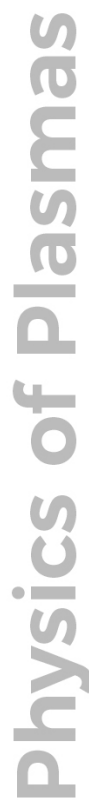

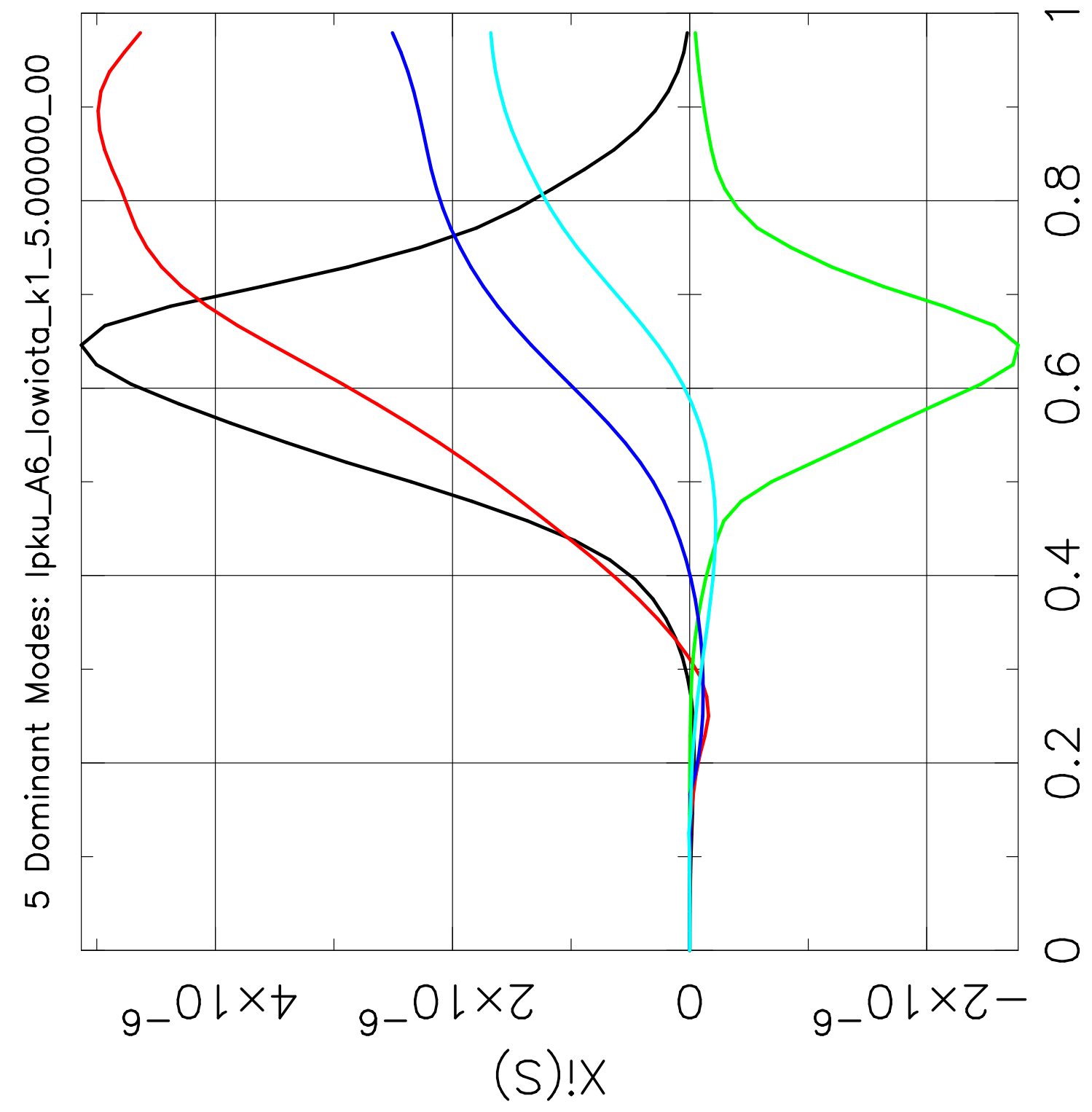

는 


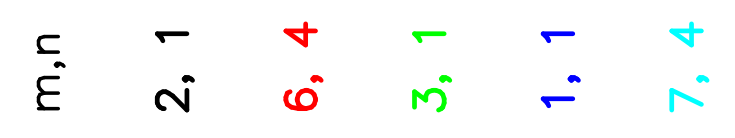

岁

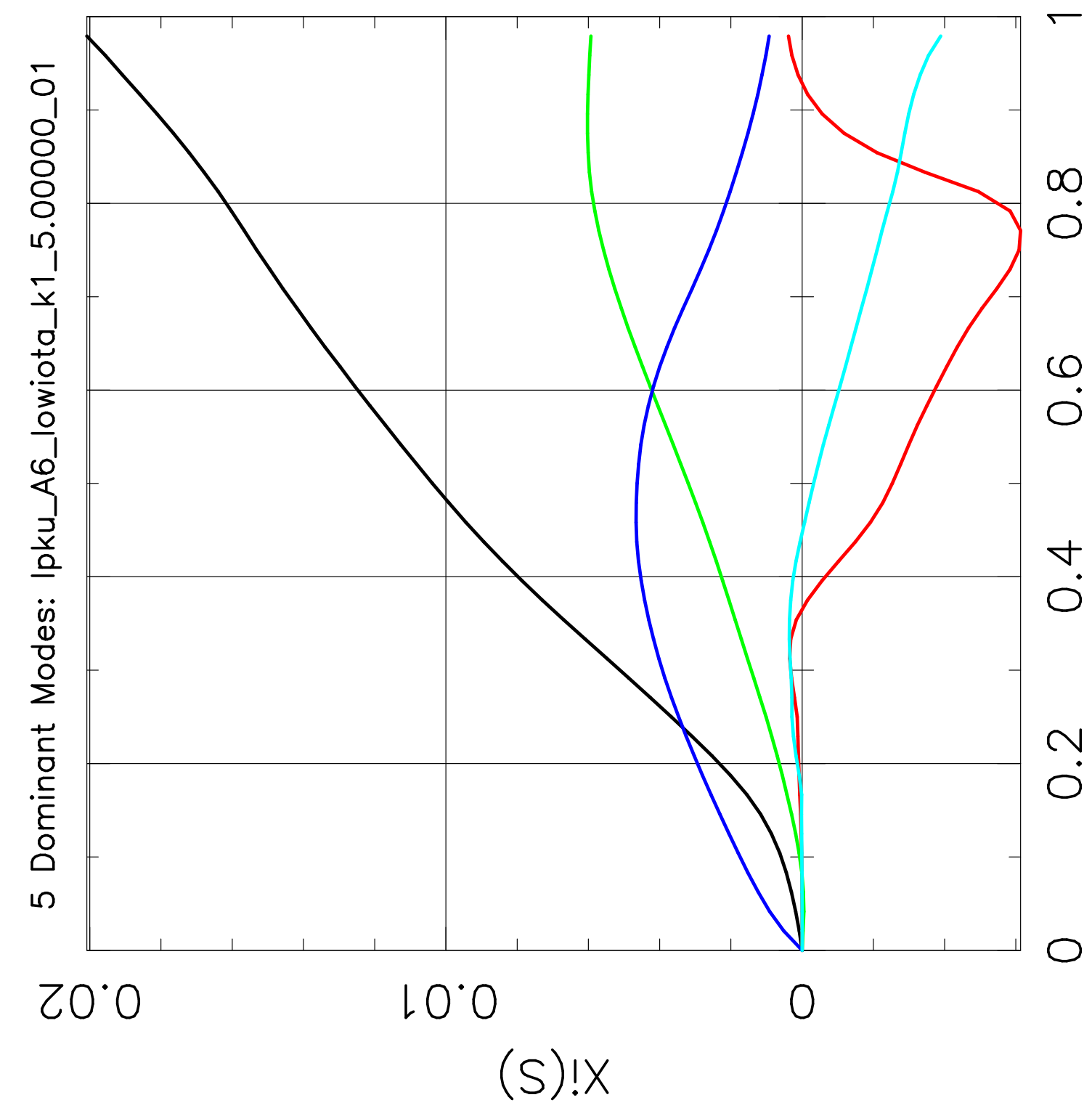




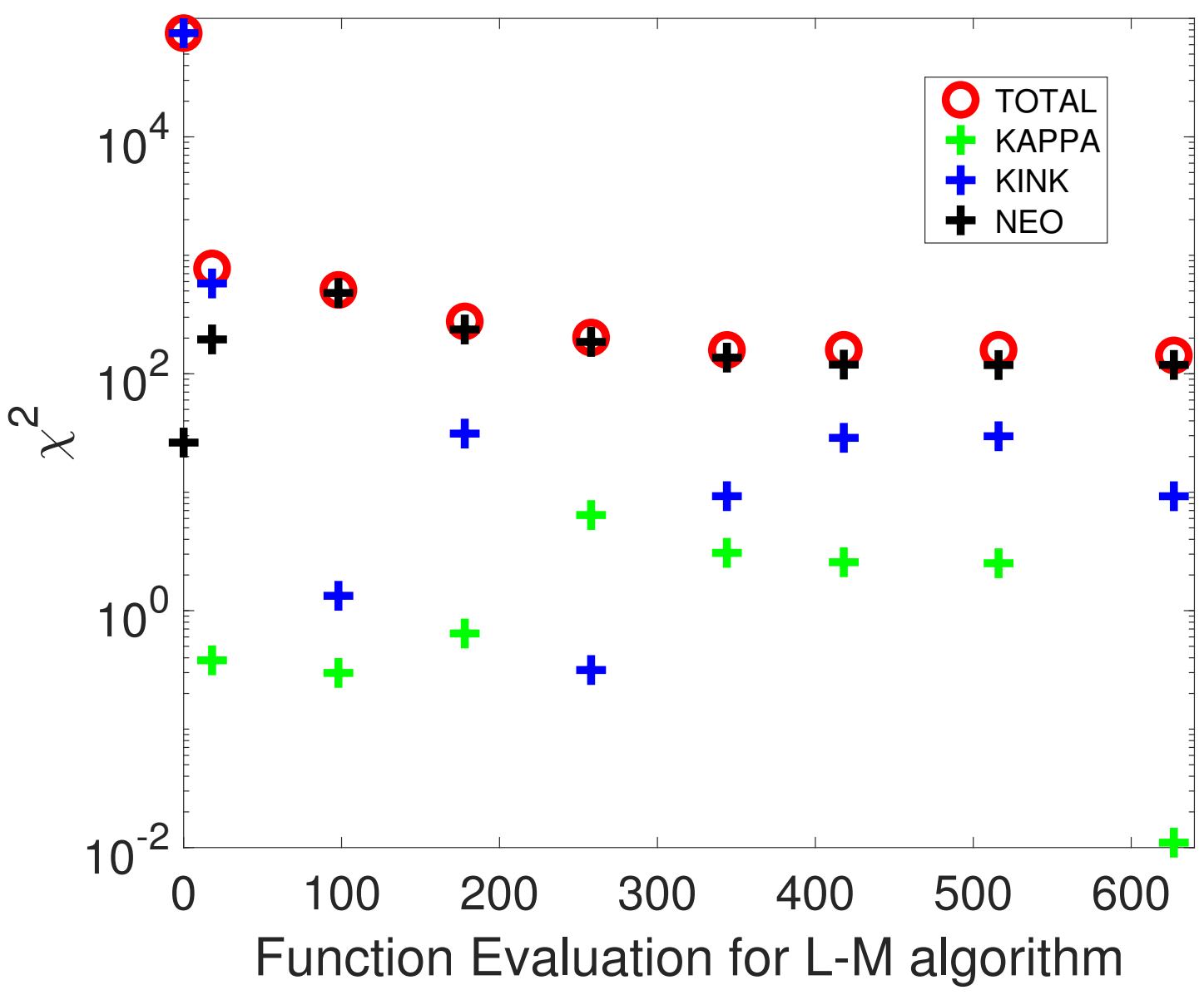




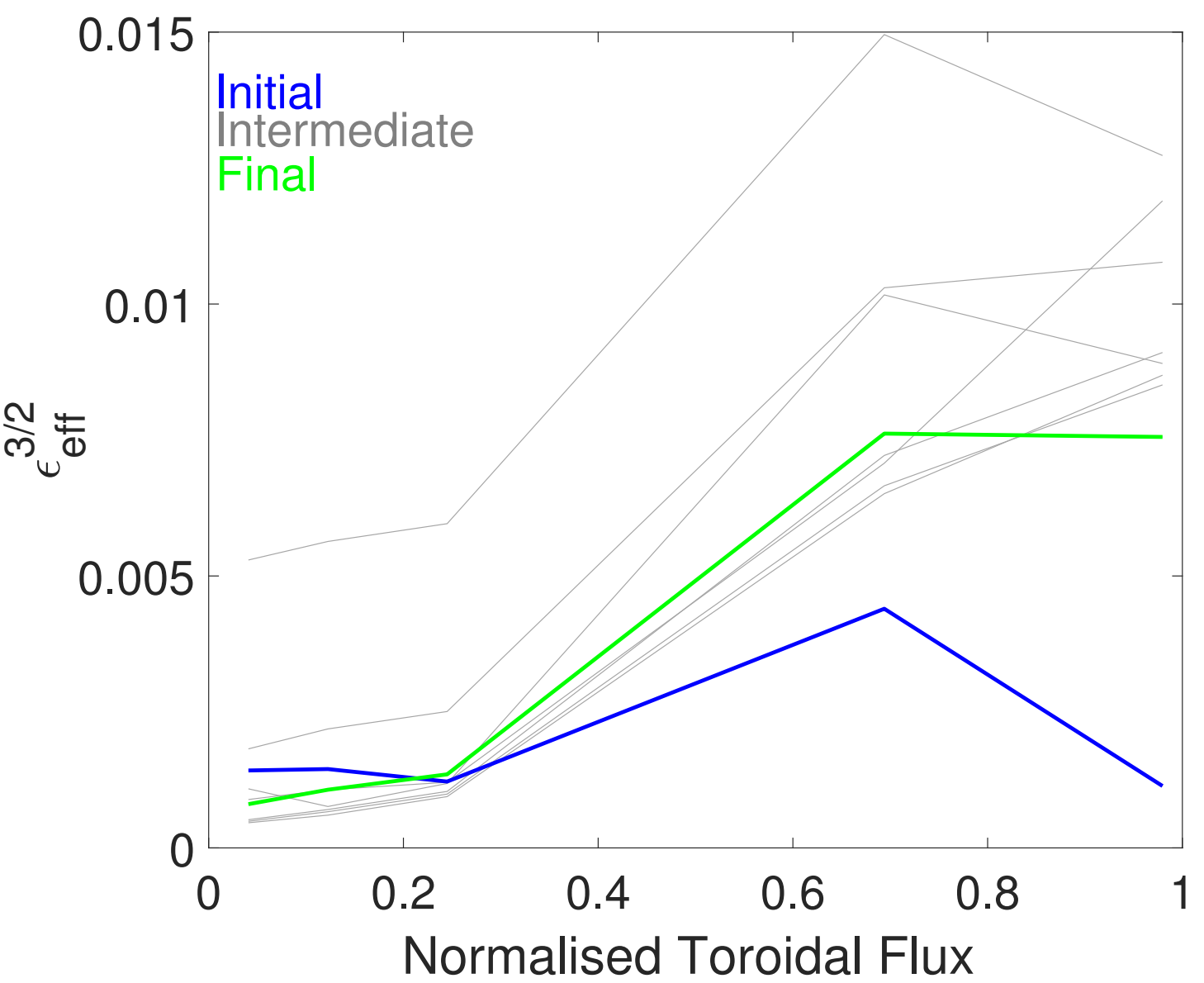




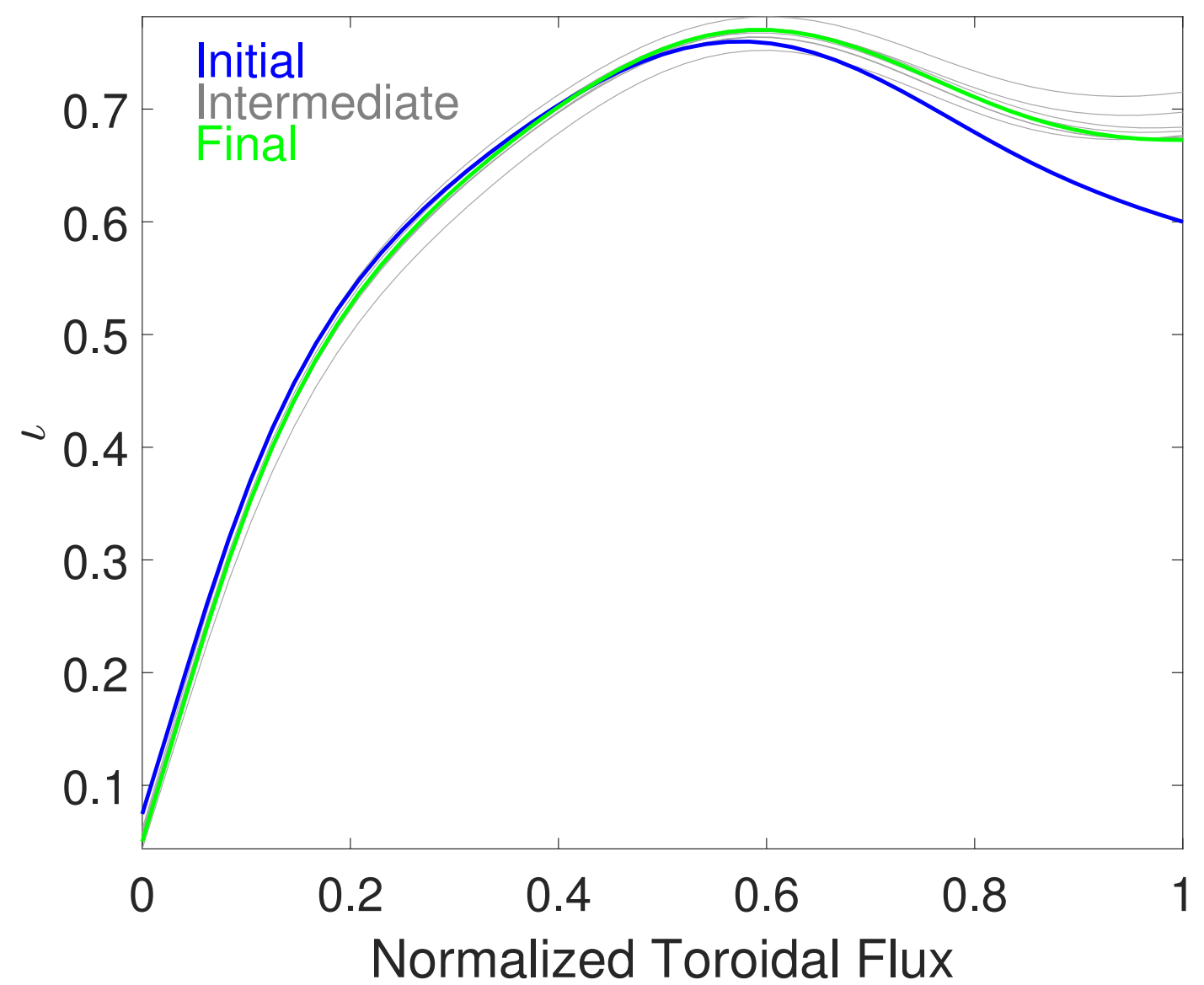



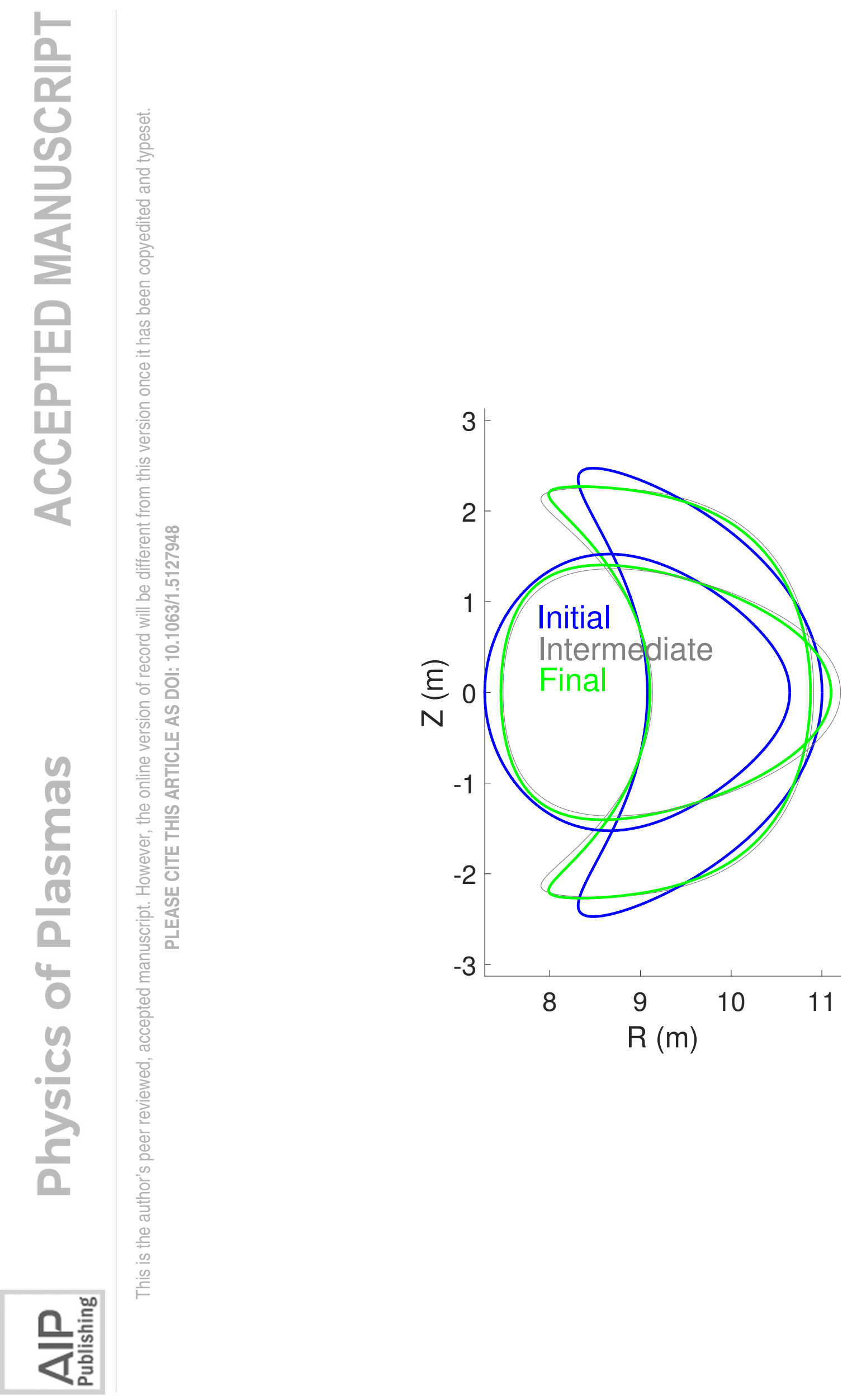


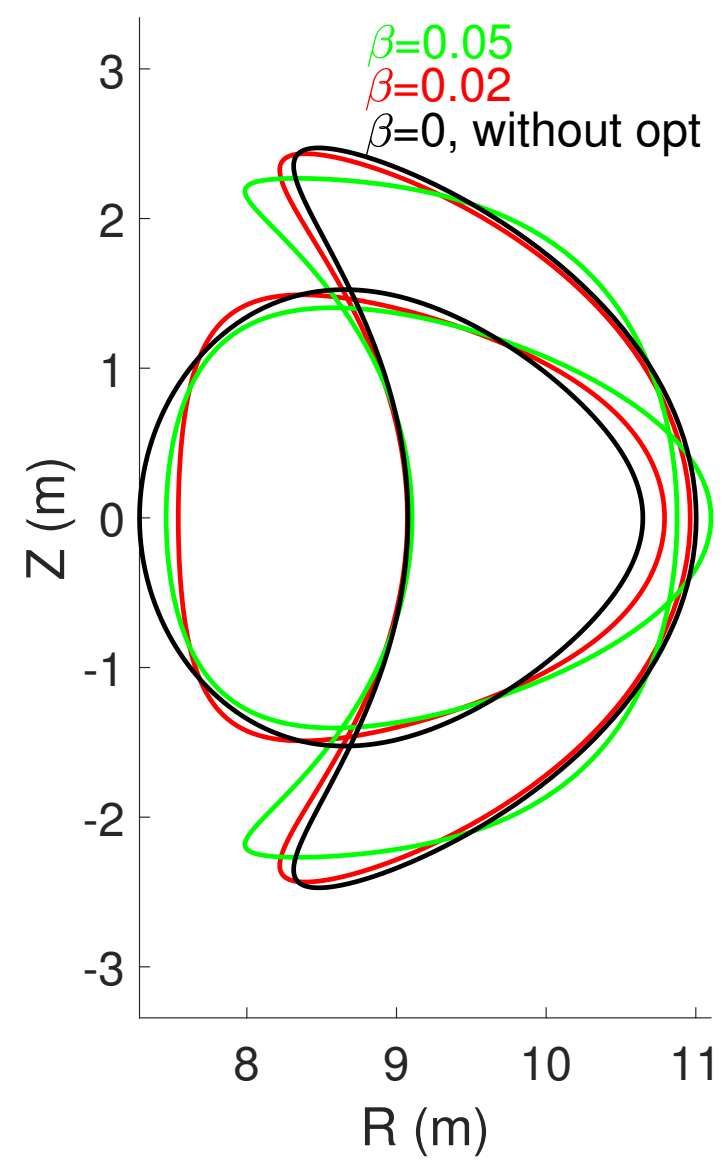

兄耪言 


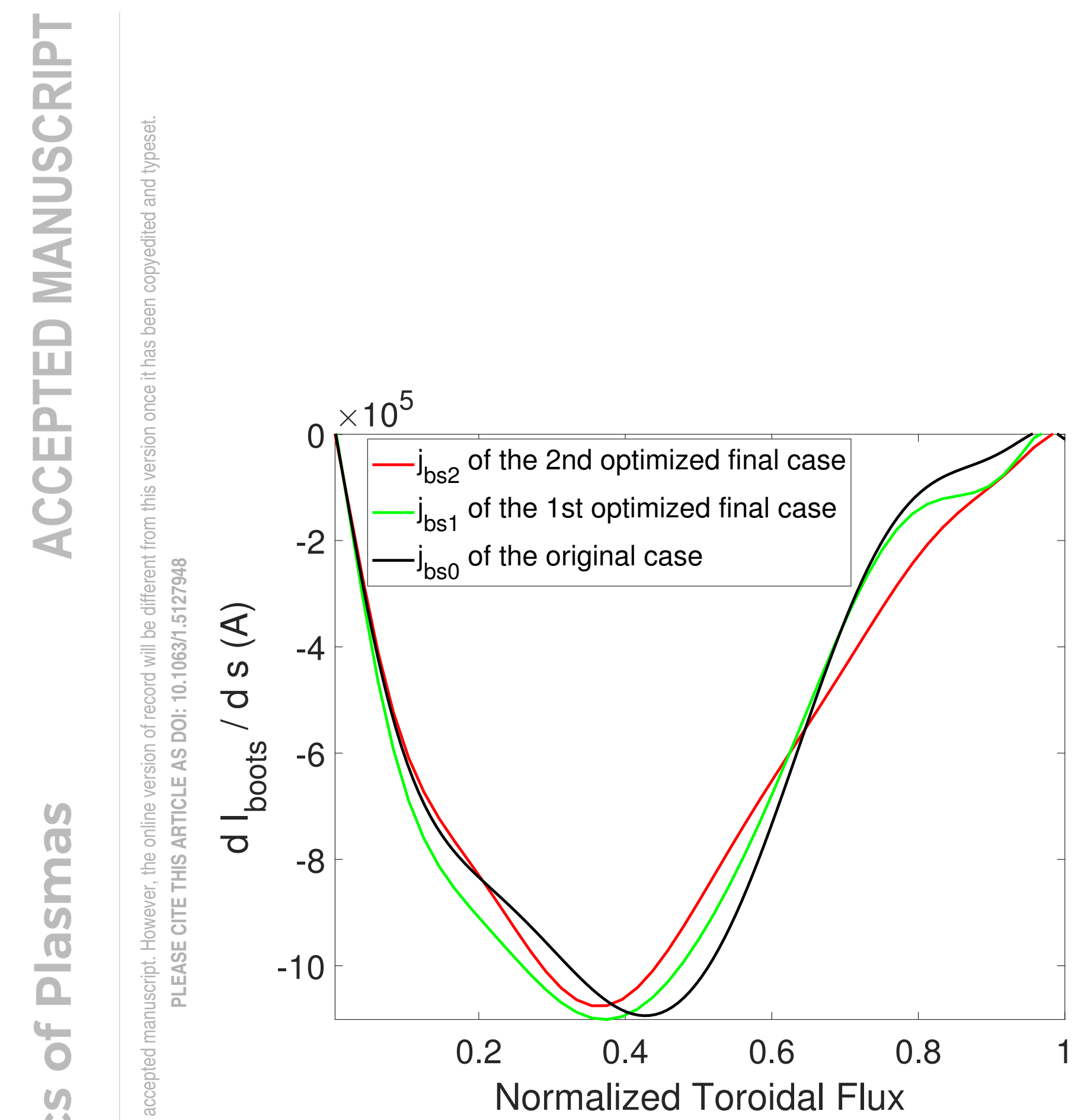




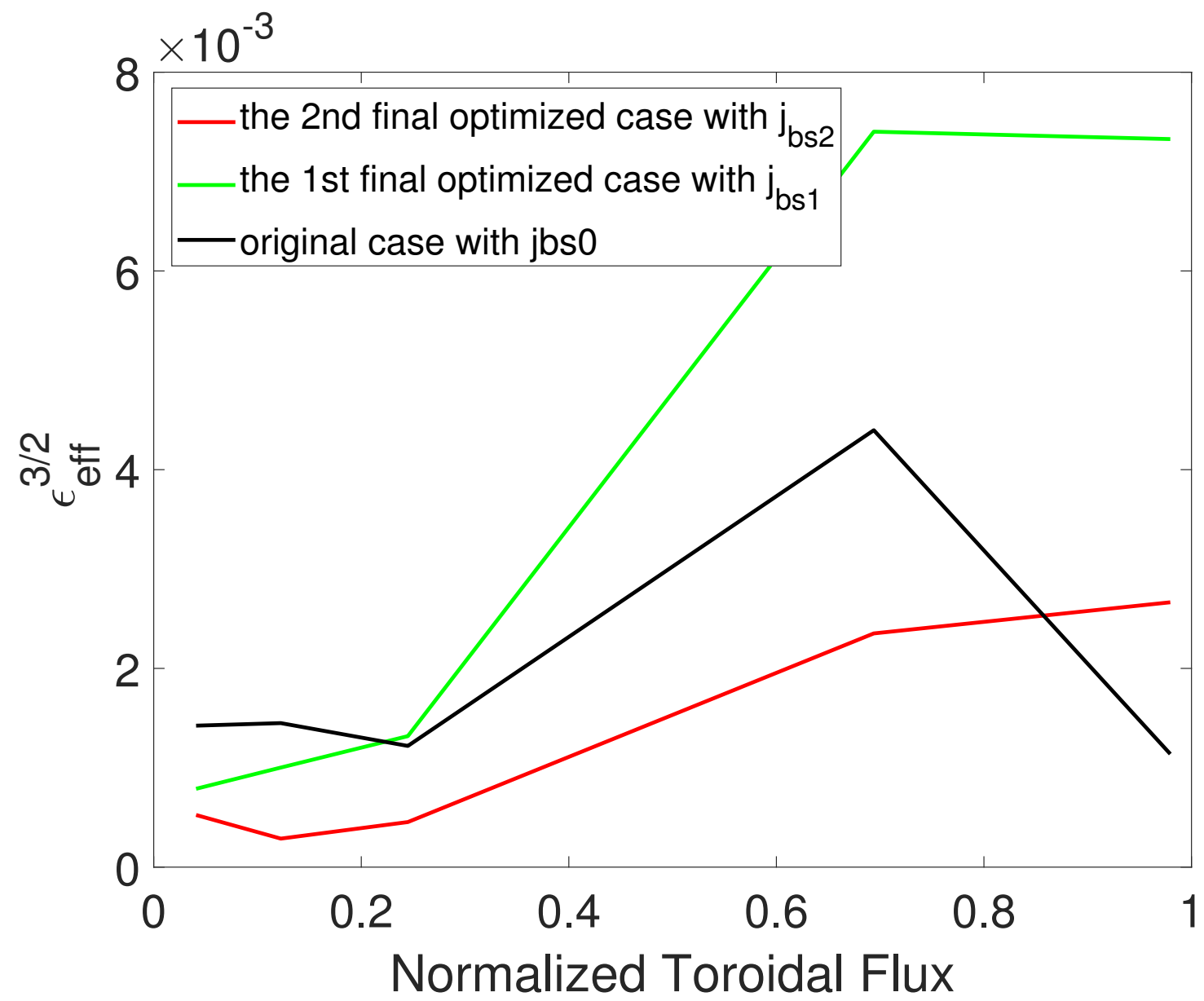




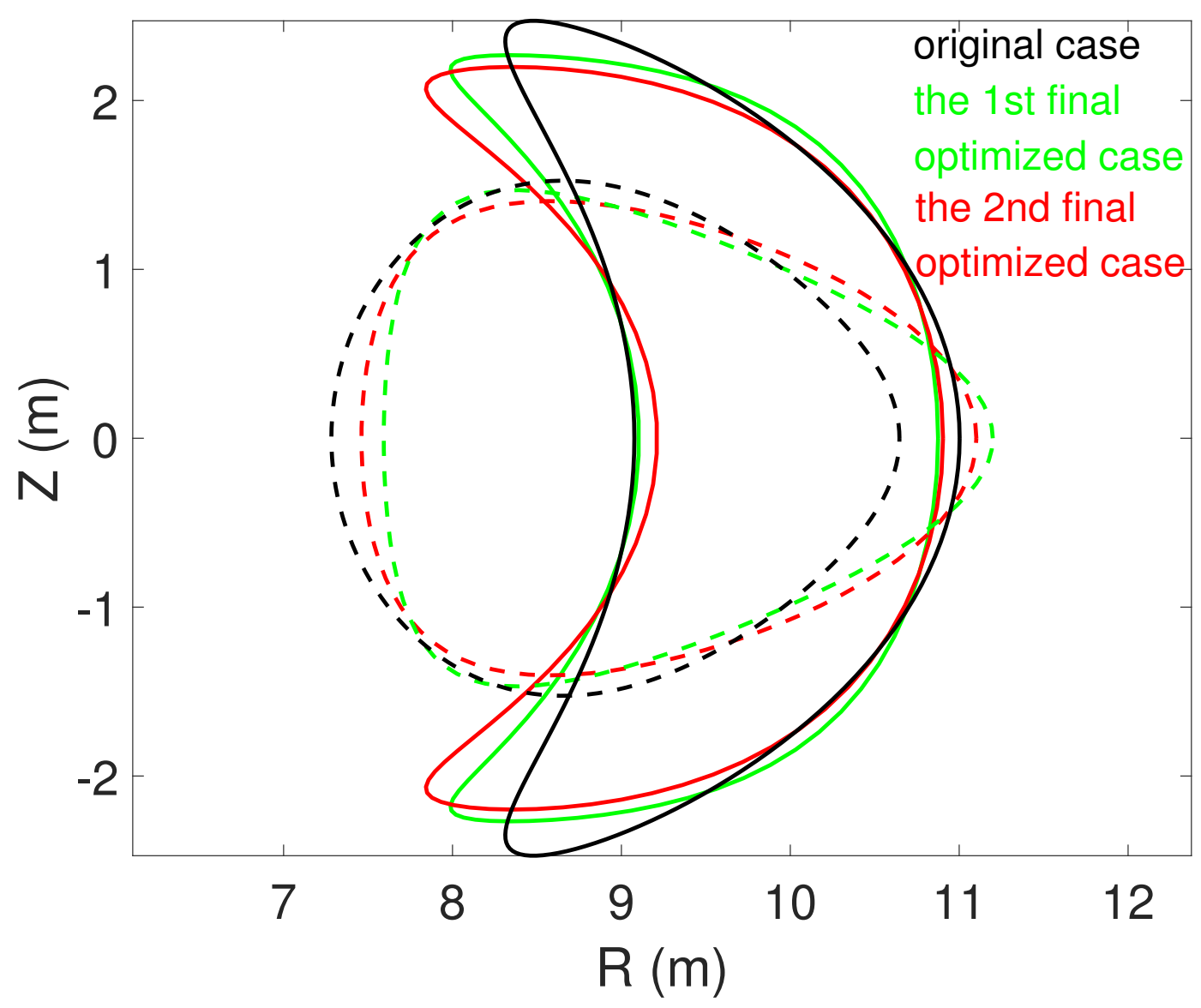

兄耪言 


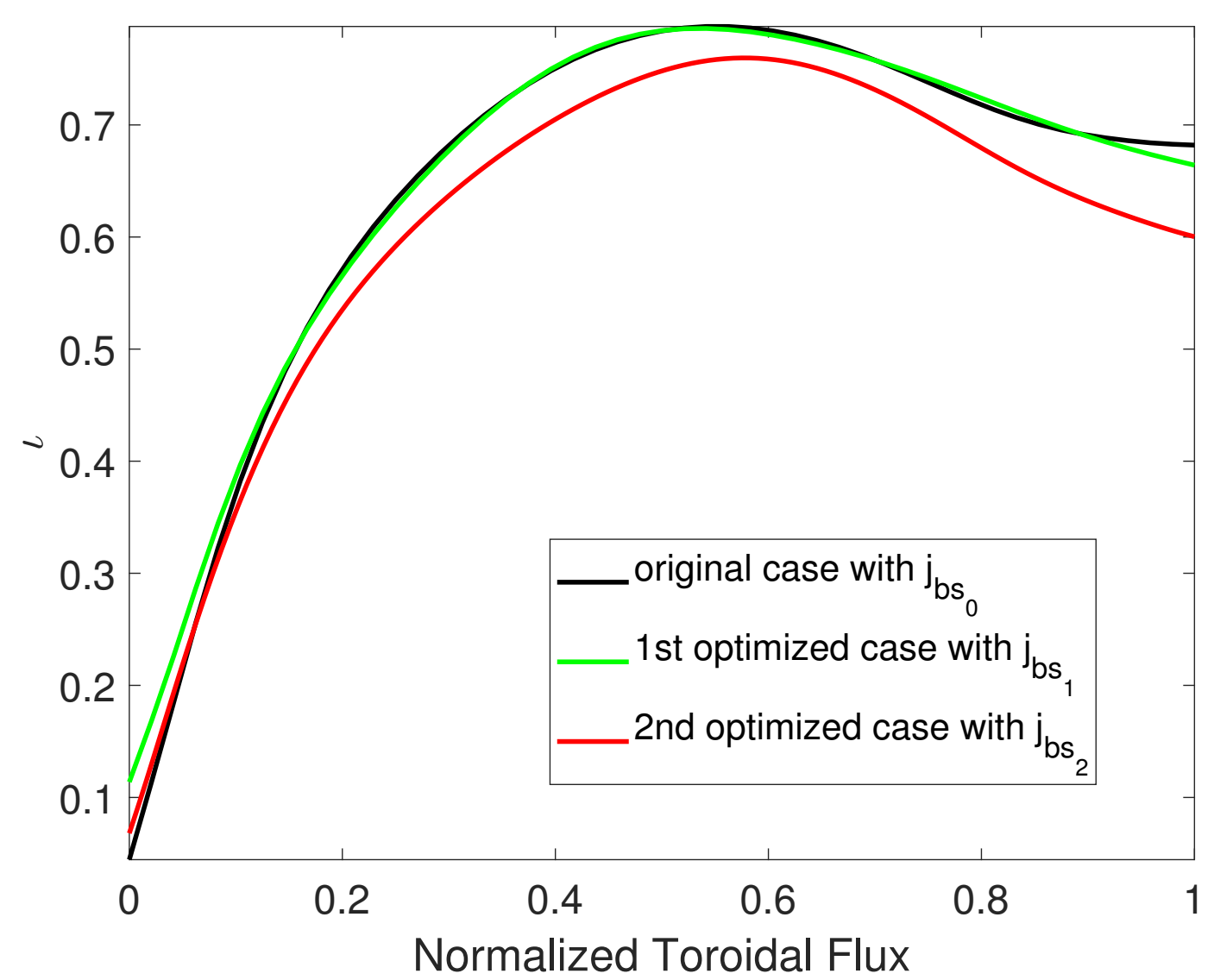

